\title{
A Technique to Classify the Similarity Solutions of Nonlinear Partial (Integro-)Differential Equations. I. Optimal Systems of Solvable Lie Subalgebras
}

\author{
H. Kötz \\ Institut für Mathematische Physik, Technische Universität Braunschweig, Braunschweig, FRG
}

Z. Naturforsch. 47 a, $1161-1173$ (1992); received July 9, 1992

\begin{abstract}
Lie group analysis is a powerful tool for obtaining exact similarity solutions of nonlinear (integro-) differential equations. In order to calculate the group-invariant solutions one first has to find the full Lie point symmetry group admitted by the given (integro-)differential equations and to determine all the subgroups of this Lie group. An effective, systematic means to classify the similarity solutions afterwards is an "optimal system", i.e. a list of group-invariant solutions from which every other such solution can be derived. The problem to find optimal systems of similarity solutions leads to that to "construct" the optimal systems of subalgebras for the Lie algebra of the known Lie point symmetry group. Our aim is to demonstrate a practicable technique for determining these optimal subalgebraic systems using the invariants relative to the group of the inner automorphisms of the Lie algebra in case of a finite-dimensional Lie point symmetry group. Here, we restrict our attention to optimal subsystems of solvable Lie subalgebras. This technique is applied to the nine-dimensional real Lie point symmetry group admitted by the two-dimensional non-stationary ideal magnetohydrodynamic equations.
\end{abstract}

Key words: Group theory; Magnetohydrodynamics; Compressible flows.

\section{Introduction}

The similarity analysis of systems of (nonlinear) partial differential equations (PDEs) leading to groupinvariant solutions is systematically and well described in the textbooks of Ovsiannikov [1], Ibragimov [2], Bluman and Cole [3], Olver [4], Bluman and Kumei [5], Stephani [6] etc. In this paper we assume that the reader is familiar with the basic tools of the similarity analysis for PDEs and knows how to find the full Lie point symmetry group admitted by the given differential equations. Nevertheless we first give a short summary of similarity analysis for a system of PDEs of first order to fix notation. Let $v, m, n$ be natural numbers and let

$$
\mathscr{F}\left(x, u, u_{x}\right):=\left(\mathscr{F}^{1}\left(x, u, u_{x}\right), \ldots, \mathscr{F}^{v}\left(x, u, u_{x}\right)\right)=0
$$

be a system of $v$ PDEs of first order with the vector $x:=\left(x^{1}, \ldots, x^{n}\right) \in \mathbb{R}^{n}$ of the $n$ independent variables, the vector $u:=\left(u^{1}, \ldots, u^{m}\right) \in \mathbb{R}^{m}$ of the $m$ dependent variables and the vector $u_{x}:=\left(\partial u^{1} / \partial x^{1}, \ldots, \partial u^{1} / \partial x^{n}\right.$, $\left.\partial u^{2} / \partial x^{1}, \ldots, \partial u^{m} / \partial x^{n}\right) \in \mathbb{R}^{m n}$ of the $n m$ derivatives.

The Lie point symmetry group $G$ (with its real Lie algebra $\mathscr{G}$ ) admitted by (1) consists of real valued local

Reprint requests to Dipl.-Phys. H. Kötz, Institut für Mathematische Physik, Mendelssohnstr. 3, W-3300 Braunschweig. transformations $g:(x, u) \mapsto g \cdot(x, u)=(\tilde{x}, \tilde{u})$, which leave the given PDEs (1) invariant and transform (graphs of) solutions to (graphs of) other solutions. The vector fields

$$
\boldsymbol{v}=\sum_{i=1}^{n} \xi^{i}(x, u) \frac{\partial}{\partial x^{i}}+\sum_{j=1}^{m} \eta^{j}(x, u) \frac{\partial}{\partial u^{j}} \in \mathscr{G}
$$

are the infinitesimal generators of $G=\exp (\mathscr{G})$, where exp: $\mathscr{G} \rightarrow G$ denotes the exponential map, which is a local diffeomorphism between $\mathscr{G}$ and $G$. The functions $\xi^{i}$ and $\eta^{j}$ are called infinitesimal elements or the coordinate functions and can be obtained by determination of the infinitesimal transformations $\tilde{x}^{i}=x^{i}+\varepsilon \xi^{i}(x, u)$, $\tilde{u}^{j}=u^{j}+\varepsilon \eta^{j}(x, u)$, which leave (1) invariant, where $\varepsilon$ denotes the real group-parameter. The Lie product $[.,]:. \mathscr{G} \times \mathscr{G} \rightarrow \mathscr{G}$ is defined as the commutator $[\boldsymbol{v}, \boldsymbol{w}]:=$ $\boldsymbol{v}(\boldsymbol{w})-\boldsymbol{w}(\boldsymbol{v})$ of the two vector fields $\boldsymbol{v}, \boldsymbol{w} \in \mathscr{G}$. For the vector field $v \in \mathscr{G}$ the corresponding group transformations $g=\exp (\varepsilon \boldsymbol{v}) \in G$ are found as the solutions of the first-order autonomous system

$$
\begin{aligned}
& \frac{d \tilde{x}^{i}}{\mathrm{~d} \varepsilon}=\xi^{i}(\tilde{x}, \tilde{u}), \quad \tilde{x}^{i}(\varepsilon=0)=x^{i} \quad(i=1, \ldots, n), \\
& \frac{\mathrm{d} \tilde{u}^{j}}{\mathrm{~d} \varepsilon}=\eta^{j}(\tilde{x}, \tilde{u}), \quad \tilde{u}^{j}(\varepsilon=0)=u^{j} \quad(j=1, \ldots, m) .
\end{aligned}
$$


In this paper we assume that the Lie point symmetry group $G$ is an $r$-parameter Lie group, where $r \in \mathbb{N}$ is a natural number. Therefore the dimension $\mathrm{d}(\mathscr{G})=$ $\operatorname{dim}(\mathscr{G})=r$ of its Lie algebra $\mathscr{G}$ is finite and there exists a basis $\left\{\boldsymbol{v}_{1}, \ldots, \boldsymbol{v}_{r}\right\}$ of $\mathscr{G}$. The structure constants $C_{j k}^{i}$ of $\mathscr{G}$ are defined by $\left[\boldsymbol{v}_{j}, \boldsymbol{v}_{k}\right]=\sum_{i=1}^{r} C_{j k}^{i} \boldsymbol{v}_{i}(i, j, k=1, \ldots, r)$. For given $\boldsymbol{v} \in \mathscr{G}$ let $\operatorname{ad}(\boldsymbol{v})$ be the endomorphism of $\mathscr{G}$ defined by $\boldsymbol{w} \mapsto \operatorname{ad}(\boldsymbol{v})(\boldsymbol{w}):=[\boldsymbol{w}, \boldsymbol{v}]^{1}$, which is termed an inner derivation (differential) of $\mathscr{G}$ or an adjoint mapping. The symmetric bilinear form $K_{\mathscr{G}}: \mathscr{G} \times \mathscr{G} \rightarrow \mathbb{R}$ defined by $K_{\mathscr{G}}(\boldsymbol{v}, \boldsymbol{w}):=\operatorname{tr}(\operatorname{ad}(\boldsymbol{v}) \circ \operatorname{ad}(\boldsymbol{w}))^{2}$ is called the (Cartan-)Killing form, which plays an important role in the structural theory of Lie algebras. The mapping ad: $\mathscr{G} \rightarrow \mathscr{D}(\mathscr{G})$ is the so-called adjoint representation of $\mathscr{G}$, where $\mathscr{D}(\mathscr{G})=\operatorname{ad}(\mathscr{G})$, i.e. the Lie algebra of all inner derivations, is called an associated algebra of the Lie algebra $\mathscr{G}$. Any $g \in G$ can be written as $g=\prod_{i=1}^{r} \exp \left(\varepsilon_{i} \boldsymbol{v}_{i}\right) \in G$.

The calculation and classification of similarity solutions is another application of Lie point symmetries. A similarity solution of a s-parameter subgroup $H \subset G$ $(s \leq r)$ is invariant for all transformations $h \in H$ and can be obtained, under some additional regularity assumptions on the action of $H$ (see Olver [4]), by solving a system of PDEs with $s$ fewer independent variables, that is called the reduced system. A function $f: \mathbb{R}^{n+m} \rightarrow \mathbb{R}$ is said to be a $H$-invariant function if $f(x, u)=f(h \cdot(x, u))$ for all $h \in H$. A complete set of functionally independent $H$-invariant functions becomes the set of new variables of the reduced system. These new variables are called similarity variables. In the most important case the reduced system is a system of ordinary differential equations (ODEs). For any $g \in G$ with $g \notin H$ a $H$-invariant solution is transformed to a $g \cdot H \cdot g^{-1}$-invariant solution. We call the two subgroups $H$ and $g \cdot H \cdot g^{-1}$ conjugate. An optimal system $\Theta_{s}^{G}$ is a list of $s$-parameter subgroups of $G$, with $s \leq r$, which contains one representative of each conjugacy class. It is sufficient to calculate the similarity solutions for the members of this optimal system, because all similarity solutions are determined by applying the symmetry transformations to the similarity solutions of the optimal system.

1 This definition follows the notation of Olver [4] or Ovsiannikov [1], although in most textbooks the mapping $\operatorname{ad}(\boldsymbol{v})$ is defined by $\operatorname{ad}(\boldsymbol{v})(\boldsymbol{w}):=[\boldsymbol{v}, \boldsymbol{w}]$.

${ }^{2} \operatorname{tr}(A)$ denotes the trace of the endomorphism $A$.
The problem of finding an optimal system $\Theta_{s}^{G}$ of $s$-parameter subgroups of $G$ is equivalent to that of finding an optimal system $\Theta_{s}^{\mathscr{G}}$ of $s$-dimensional Lie subalgebras of $\mathscr{G}$ (see Olver [4]), where two subalgebras $\mathscr{H}$ and $\mathscr{K}$ are called conjugate if there exists a $g \in G$ such that $\mathscr{K}=\operatorname{Ad}(g)(\mathscr{H})$. $\operatorname{Ad}(g)$ denotes an inner automorphism, which is a linear mapping on $\mathscr{G}$. For $g=\exp (\varepsilon \boldsymbol{v})$ with the real parameter $\varepsilon$ and $\boldsymbol{v} \in \mathscr{G}$ it maps $\boldsymbol{w} \in \mathscr{G}$ to

$$
\begin{aligned}
\operatorname{Ad}(\exp (\varepsilon \boldsymbol{v}))(\boldsymbol{w}) & =\exp (\varepsilon \operatorname{ad}(\boldsymbol{v}))(\boldsymbol{w})=\sum_{j=0}^{\infty} \frac{\varepsilon^{j}}{j !}(\operatorname{ad}(\boldsymbol{v}))^{j}(\boldsymbol{w}) \\
& =\boldsymbol{w}+\varepsilon[\boldsymbol{w}, \boldsymbol{v}]+\frac{\varepsilon^{2}}{2}[[\boldsymbol{w}, \boldsymbol{v}], \boldsymbol{v}]+\ldots
\end{aligned}
$$

The local Lie group Int $(\mathscr{G})$ consists of all inner automorphisms of $\mathscr{G}$. Ad: $G \rightarrow \operatorname{Int}(\mathscr{G}), g \mapsto \operatorname{Ad}(g)$ is called the adjoint representation of $G$. The difficult algebraic problem of obtaining optimal subalgebraic systems has not yet been completely solved in the sense of the formation of a definite algorithm according to which the optimal systems $\Theta_{s}^{\mathscr{G}}$ can be "constructed". The determination of optimal systems can be done relatively easily only for small dimensionality. Thus the theory for constructing optimal systems of Lie subalgebras needs to be developed further, as Ovsiannikov [1] notices. Both Ovsiannikov [1] and Olver [4] make use of the invariance property of the Killing form, that is an invariant relative to $\operatorname{Int}(\mathscr{G})$, to determine optimal systems of one-dimensional subalgebras in special examples. Our idea is to use all functionally independent symmetric bilinear forms which are invariant relative to the considered Lie group of inner automorphisms and their subgroups to simplify and to shorten the determination of optimal subalgebraic systems for small dimensionality. In Sect. 1 we give a short summary of the known techniques to find optimal systems of solvable subalgebras of a Lie algebra $\mathscr{G}$, which can be found in the textbooks of Ovsiannikov [1], Ibragimov [2] and Olver [4]. In addition we describe our further developments of these techniques based on the computer-aided calculation of the bilinear invariants for the regarded inner automorphisms.

In Sect. 2 we demonstrate the advantage of our technique for constructing optimal subalgebraic systems in case of small dimensionality by applying it to the finite-dimensional solvable subalgebra of the Lie algebra of infinitesimal symmetries admitted by the two-dimensional non-stationary ideal magnetohydrodynamic (MHD) equations. In comparison with the 
usual techniques to determine an optimal system of one-dimensional subalgebras (see Galas [7]), the usage of our modified technique for obtaining the same optimal system saves a lot of time.

\section{Optimal Systems of Solvable Lie Subalgebras}

In this section we summarize the details for "constructing" optimal systems of solvable subalgebras for the real Lie algebra $\mathscr{G}$ of a given $r$-parameter Lie point symmetry group $G$, where $r$ is a natural number. The summary is based on the textbooks of Ovsiannikov [1], § 13, § 14, § 17 and Ibragimov [2], § 2, which Galas [7] used to formulate an "algorithm" for determining optimal subalgebraic systems without using the invariants of the group of the inner automorphisms. The proofs of the below stated theorems can be found in most books on Lie groups and Lie algebras, for example Jacobson [8], Sagle and Walde [9] or Varadarajan [10]. We denote the dimension of $\mathscr{G}$ by $\mathrm{d}(\mathscr{G})=r$ and suppose that the set $\left\{\boldsymbol{v}_{1}, \ldots, \boldsymbol{v}_{r}\right\}$ is a basis of $\mathscr{G}$. In the following, $\mathscr{H}\left(\boldsymbol{u}_{1}, \ldots, \boldsymbol{u}_{\boldsymbol{s}}\right)$ denotes the subalgebra $\mathscr{H}$ of $\mathscr{G}$ with the basis $\left\{\boldsymbol{u}_{1}, \ldots, \boldsymbol{u}_{s}\right\}$ (this notation follows Ovsiannikov [1]). As mentioned above, an optimal system $\Theta_{s}^{\mathscr{G}}$ of $s$-dimensional subalgebras of $\mathscr{G}$ with $s \leq \mathrm{d}(\mathscr{G})=r$ is the union of the representatives of conjugate (or similar) algebra classes of given dimensionality $s$ (one from every class), where two $s$-dimensional subalgebras $\mathscr{H}\left(\boldsymbol{u}_{1}, \ldots, \boldsymbol{u}_{s}\right)$ and $\mathscr{K}\left(\boldsymbol{w}_{1}, \ldots, \boldsymbol{w}_{s}\right)$ are called conjugate if there exists an inner automorphism $\operatorname{Ad}(g) \in \operatorname{Int}(\mathscr{G})(g \in G)$ such that

$$
\left(\begin{array}{c}
\operatorname{Ad}(g)\left(\boldsymbol{u}_{1}\right) \\
\vdots \\
\operatorname{Ad}(g)\left(\boldsymbol{u}_{s}\right)
\end{array}\right)=\left(\begin{array}{ccc}
a_{11} & \ldots & a_{1 s} \\
\vdots & & \vdots \\
a_{s 1} & \ldots & a_{s s}
\end{array}\right)\left(\begin{array}{c}
\boldsymbol{w}_{1} \\
\vdots \\
\boldsymbol{w}_{s}
\end{array}\right)
$$

with

$$
\left(\begin{array}{lll}
a_{11} & \ldots & a_{1 s} \\
\vdots & & \vdots \\
a_{s 1} & \ldots & a_{s s}
\end{array}\right) \in \operatorname{GL}(s ; \mathbb{R}),
$$

i.e. $\operatorname{Ad}(g)(\mathscr{H})=\mathscr{K}$. The common inner automorphism in the Lie group $\operatorname{Int}(\mathscr{G})$ is

$$
\begin{aligned}
\operatorname{Ad}(g & \left.=\exp \left(\varepsilon_{1} \boldsymbol{v}_{1}\right) \cdot \ldots \cdot \exp \left(\varepsilon_{\boldsymbol{r}} \boldsymbol{v}_{r}\right)\right) \\
& =\operatorname{Ad}\left(\exp \left(\varepsilon_{1} \boldsymbol{v}_{1}\right)\right) \circ \ldots \circ \boldsymbol{A d}\left(\exp \left(\varepsilon_{r} \boldsymbol{v}_{r}\right)\right),
\end{aligned}
$$

where $\operatorname{Ad}\left(g=\exp \left(\varepsilon_{j} \boldsymbol{v}_{j}\right)\right)$ is defined in (3) (see Ovsiannikov [1]). In case of $s=1$ the two one-dimensional Lie subalgebras $\mathscr{H}(\boldsymbol{u}) \subset \mathscr{G}$ and $\mathscr{K}(\boldsymbol{w}) \subset \mathscr{G}$ with the fixed basis vectors $\boldsymbol{u}=\sum_{j=1}^{r} \alpha_{j} \boldsymbol{v}_{j}$ and $\boldsymbol{w}=\sum_{j=1}^{r} \beta_{j} \boldsymbol{v}_{j}\left(\alpha_{j}, \beta_{j} \in \mathbb{R}\right.$; $j=1, \ldots, r)$ are conjugate if and only if there exist an $\operatorname{Ad}(g) \in \operatorname{Int}(\mathscr{G})$ and a real number $a \neq 0$, such that $\boldsymbol{w}=(1 / a) \operatorname{Ad}(g)(\boldsymbol{u})$.

The techniques for determining optimal saubalgebraic systems $\Theta_{s}^{\mathscr{G}}$ depend on the solvability of the $r$-dimensional Lie algebra $\mathscr{G}$ in case of $s \geq 3(s \leq r)$. A Lie algebra $\mathscr{G}$ is said to be solvable, if the series of ideals ${ }^{3} \mathscr{G}^{(0)}:=\mathscr{G}, \mathscr{G}^{(k+1)}:=\left[\mathscr{G}^{(k)}, \mathscr{G}^{(k)}\right]$ with $k=$ $0,1,2, \ldots$, which is called the derived series of $\mathscr{G}$, terminates, i.e. $\mathscr{G}^{\left(k_{0}\right)}=0$ for some $k_{0}$. Since one- and twodimensional Lie algebras are solvable, the optimal systems $\Theta_{1}^{\mathscr{G}}$ and $\Theta_{2}^{\mathscr{G}}$ for any Lie algebra $\mathscr{G}$ consist of conjugacy classes of solvable subalgebras. Instead of the above definition one can use the following criterion of solvability for $\mathscr{G}$ :

Cartan's Criterion of Solvability (see e.g. Ovsiannikov [1]). A finite-dimensional Lie algebra $\mathscr{G}$ is solvable if and only if $K_{\mathscr{G}}(\boldsymbol{u}, \boldsymbol{u}):=\operatorname{tr}(\operatorname{ad}(\boldsymbol{u}) \circ \operatorname{ad}(\boldsymbol{u}))=0$ for any $\boldsymbol{u} \in \mathscr{G}^{(1)}$, where $\mathscr{G}^{(1)}=[\mathscr{G}, \mathscr{G}]$ is called the derived algebra of $\mathscr{G}$.

At this the Killing form $K_{\mathscr{G}}: \mathscr{G} \times \mathscr{G} \rightarrow \mathbb{R}$ maps $(\boldsymbol{u}, \boldsymbol{w}) \in \mathscr{G} \times \mathscr{G}$ with $\boldsymbol{u}=\sum_{j=1}^{r} \alpha_{j} \boldsymbol{v}_{j}, \boldsymbol{w}=\sum_{j=1}^{r} \beta_{j} \boldsymbol{v}_{j}$ to

$$
K_{\mathscr{G}}(\boldsymbol{u}, \boldsymbol{w}):=\operatorname{tr}(\mathbf{a d}(\boldsymbol{u}) \circ \mathbf{a d}(\boldsymbol{w}))=\sum_{i, j=1}^{r} \alpha_{i} K_{i j}^{\mathscr{G}} \beta_{j}
$$

with the real symmetric matrix

$$
K^{\mathscr{G}}:=\left(K_{i j}^{\mathscr{G}}\right)=\left(\sum_{k, l=1}^{r} C_{i l}^{k} C_{k j}^{l}\right),
$$

which we call the Killing matrix. For a solvable Lie algebra $\mathscr{G}$ the following important theorems hold:

Lemma 1 (see e.g. Ovsiannokov [1]). Any subalgebra $\mathscr{H}$ of a solvable Lie algebra $\mathscr{G}$ is solvable. For any ideal $\mathscr{I}$ of a solvable Lie algebra $\mathscr{G}$ the factor algebra $\mathscr{G} / \mathscr{I}$ is solvable. If $\mathscr{G}$ contains a solvable ideal $\mathscr{I}$ such that the factor algebra $\mathscr{G} / \mathscr{I}$ is solvable, then $\mathscr{G}$ is also solvable.

Lie Theorem (see e.g. Varadarajan [10]). For any $r$-dimensional solvable real Lie algebra $\mathscr{G}$ there exists a

${ }^{3}$ Any subalgebra $\mathscr{H}$ of $\mathscr{G}$ is said to be an ideal of $\mathscr{G}$ if it satisfies $[\mathscr{G}, \mathscr{H}] \subset \mathscr{H}$. The null algebra 0 , which consists of the zero vector, and $\mathscr{G}$ are called the trivial ideals. All remaining ideals of $\mathscr{G}$ are called the proper ideals of $\mathscr{G}$ (see e.g. Ovsiannikov [1]). 
chain of subalgebras

$$
\mathscr{G}=: \mathscr{G}_{r} \supset \mathscr{G}_{r-1} \supset \ldots \supset \mathscr{G}_{1} \supset \mathscr{G}_{0}:=0
$$

such that $\mathscr{G}_{j-1}$ is an ideal of $\mathscr{G}_{j}$ and $\operatorname{dim}\left(\mathscr{G}_{j}\right)=j$ for $1 \leq j \leq r$.

With the help of the invariants of the group of the inner automorphisms, which are considered in subsection 1.1, it is possible to shorten the lengthy "construction" of optimal systems. Especially the detection of the invariant linear and (symmetric) bilinear forms of the Lie algebra $\mathscr{G}$ and of its subalgebras is helpful during the classification process. They can be calculated by our REDUCE 3.2 programs OPTSYS and BINV, which are briefly described in subsection 1.3. In subsection 1.2 we describe the "construction" of $\Theta_{1}^{\mathscr{G}}$, $\Theta_{2}^{\mathscr{G}}$ for a not necessarily solvable Lie algebra $\mathscr{G}$ and of the optimal subsystems $\Theta_{s}^{\mathscr{G}}$ of solvable subalgebras for an $r$-dimensional Lie algebra $\mathscr{G}$ for $3 \leq s \leq r$. The latter are the full optimal subalgebraic systems if $\mathscr{G}$ is solvable.

For an $r$-dimensional non solvable Lie algebra $\mathscr{G}$, methods to determine full optimal systems $\Theta_{s}^{\mathscr{G}}$ with $3 \leq s \leq r$ will be presented in a following paper.

\subsection{Invariants of the Inner Automorphisms}

In "constructing" the optimal subalgebraic systems of the $r$-dimensional Lie algebra $\mathscr{G}$, it is useful to know the scalar invariants for the group of the inner automorphisms Int $(\mathscr{G})$ of $\mathscr{G}$, where a complex valued function $\chi: \mathscr{G} \rightarrow \mathbb{C}$ is called invariant if $\chi(\operatorname{Ad}(g)(\boldsymbol{u}))=\chi(\boldsymbol{u})$ holds for any $\boldsymbol{u} \in \mathscr{G}$ and any inner automorphism $\operatorname{Ad}(g) \in \operatorname{Int}(\mathscr{G})$. With the help of these invariants all the vector fields $\boldsymbol{u} \in \mathscr{G}$ can be separated into nonintersecting classes. Among these invariants the linear and bilinear ones, which correspond to invariant linear and (symmetric) bilinear forms, respectively, play a special role. They can be determined by a simple "algorithm", and in many cases all the scalar invariants relative to Int $(\mathscr{G})$ can be derived from a complete set of functionally independent linear and bilinear invariants.

A linear form

$$
\varphi: \mathscr{G} \rightarrow \mathbb{R} ; \boldsymbol{w}:=\sum_{j=1}^{r} \beta_{j} \boldsymbol{v}_{j} \mapsto \varphi(\boldsymbol{w}):=\sum_{j=1}^{r} \tilde{\varphi}_{j} \beta_{j}
$$

with the real coefficients $\tilde{\varphi}_{j}(j=1, \ldots, r)$ is called invariant (relative to the group $\operatorname{Int}(\mathscr{G})$ ) if

$$
\varphi(\operatorname{Ad}(g)(\boldsymbol{w}))=\varphi(\boldsymbol{w})
$$

holds for any inner automorphism $\operatorname{Ad}(g) \in \operatorname{Int}(\mathscr{G})$ and for any $\boldsymbol{w} \in \mathscr{G}$. If $\varphi$ is an invariant linear form and $\boldsymbol{u}$, $\boldsymbol{w}$ are conjugate vector fields, then

$$
\varphi\left(\boldsymbol{w}=\frac{1}{a} \operatorname{Ad}(g)(\boldsymbol{u})\right)=\frac{1}{a} \varphi(\operatorname{Ad}(g)(\boldsymbol{u}))=\frac{1}{a} \varphi(\boldsymbol{u})
$$

holds for a proper inner automorphism $\operatorname{Ad}(g) \in \operatorname{Int}(\mathscr{G})$ and a proper nonzero $a \in \mathbb{R}$. After rescaling of $\boldsymbol{w}$, i.e. we choose $a=1$, the values of $\varphi(\boldsymbol{w})$ and $\varphi(\boldsymbol{u})$ are equal. An infinitesimal criterion of invariance of a linear form $\varphi: \mathscr{G} \rightarrow \mathbb{R}$ is

$$
\begin{aligned}
& \varphi(\operatorname{ad}(\boldsymbol{u})(\boldsymbol{w}))= \sum_{j=1}^{r}\left(\sum_{k, l=1}^{r} \beta_{k} C_{k l}^{j} \alpha_{l}\right) \tilde{\varphi}_{j}=0 \\
& \text { for any } \quad \boldsymbol{u}:=\sum_{l=1}^{r} \alpha_{l} \boldsymbol{v}, \quad \boldsymbol{w}:=\sum_{k=1}^{r} \beta_{k} \boldsymbol{v}_{k} \in \mathscr{G},
\end{aligned}
$$

which follows from (6) by choosing $g=\exp (\varepsilon \boldsymbol{u})$ and differentiating it with respect to $\varepsilon$ at the point $\varepsilon=0$. Here the adjoint mapping $\operatorname{ad}(\boldsymbol{u})$ for $\boldsymbol{u}=\sum_{l=1}^{r} \alpha_{l} \boldsymbol{v}_{l} \in \mathscr{G}$ can be represented by the matrix $\left(\sum_{l=1}^{r} C_{k l}^{j} \alpha_{l}\right)$, where $j$ indicates the row and $k$ the column. Since the coefficients $\alpha_{l}$ of $\boldsymbol{u}$ and $\beta_{k}$ of $\boldsymbol{w}$ are arbitrary real numbers, (7) can be replaced by the following overdetermined homogeneous system of $r^{2}$ linear equations:

$$
\sum_{k=1}^{r} C_{i j}^{k} \tilde{\varphi}_{k}=0 \text { for all } i, j=1, \ldots, r .
$$

All the vectors $\boldsymbol{u} \in \mathscr{G}$ are separated into the two nonintersecting classes $\varphi(\boldsymbol{u}) \neq 0$ and $\varphi(\boldsymbol{u})=0$ for any invariant linear form $\varphi \neq \equiv$, where the set $\mathscr{H}_{\varphi}:=$ $\{\boldsymbol{w} \in \mathscr{G} \mid \varphi(\boldsymbol{w})=0\}$ forms an ideal of $\mathscr{G}$. The common invariant linear form $\varphi^{\mathscr{G}}: \mathscr{G} \rightarrow \mathbb{R}$ for $\mathscr{G}$ can be determined with the help of computer algebra from criterion (7). If a nonzero invariant linear form $\varphi^{\mathscr{G}}$ exists which depends on some free real numbers, say $a_{1}, \ldots, a_{p} \in \mathbb{R}(p \in \mathbb{N} ; p \leq r)$, there is a decomposition $\varphi^{\mathscr{G}}=\sum_{j=1}^{p} a_{j} \varphi^{(j)}$ such that the invariant linear forms $\varphi^{(1)}, \ldots, \varphi^{(p)}$ have fixed real coefficients $\tilde{\varphi}_{i}^{(j)}(i=1, \ldots, r$; $j=1, \ldots, p)$. Then we consider the expression $\varphi^{\mathscr{G}}(\boldsymbol{u})=$ $\sum_{j=1}^{p} a_{j} \varphi^{(j)}(\boldsymbol{u})$ and call the expressions $\varphi^{(1)}(\boldsymbol{u}), \ldots, \varphi_{r}^{(p)}(\boldsymbol{u})$ the linear invariants of the vector field $\boldsymbol{u}=\sum_{j=1}^{r} \alpha_{j} \boldsymbol{v}_{j}$ (relative to the group Int $(\mathscr{G})$ ). During the determination of linear invariants of a vector $\boldsymbol{u} \in \mathscr{G}$ a special role is played by the center of the Lie algebra $\mathscr{G}$

$$
\mathscr{Z}(\mathscr{G}):=\{\boldsymbol{w} \in \mathscr{G} \mid \operatorname{ad}(\boldsymbol{v})(\boldsymbol{w})=[\boldsymbol{w}, \boldsymbol{v}]=0 \forall \boldsymbol{v} \in \mathscr{G}\},
$$


since every vector $\boldsymbol{w} \in \mathscr{Z}(\mathscr{G})$ is invariant relative to any inner automorphism in $\operatorname{Int}(\mathscr{G})$. If $\mathscr{G}$ has a nonnull center $\mathscr{Z}(\mathscr{G})$, which is an ideal of $\mathscr{G}$, and there exists a decomposition $\mathscr{G}=\mathscr{Z}(\mathscr{G}) \oplus \mathscr{G}$, we choose a basis $\left\{\boldsymbol{v}_{1}, \ldots, \boldsymbol{v}_{\mathbf{r}}\right\}$ of $\mathscr{G}$ such that the subalgebra $\widetilde{\mathscr{G}} \subset \mathscr{G}$ and the center $\mathscr{Z}(\mathscr{G})$ are spanned by the vectors $\boldsymbol{v}_{1}, \ldots, \boldsymbol{v}_{\tilde{r}}$ and $\boldsymbol{v}_{\tilde{r}+1}, \ldots, \boldsymbol{v}_{r}$, respectively. Then the nonzero coefficients $\alpha_{\tilde{r}+1}, \ldots, \alpha_{r}$ of $\boldsymbol{u}=\sum_{j=1}^{r} \alpha_{j} \boldsymbol{v}_{j} \in \mathscr{G}$ are linear invariants of $\boldsymbol{u}$ (see e.g. Ovsiannikov [1]). Another example for a linear invariant of an arbitrary $\boldsymbol{u}=\sum_{j=1}^{r} \alpha_{j} \boldsymbol{v}_{j} \in \mathscr{G}$ is the trace $\operatorname{tr}(\operatorname{ad}(u))=\sum_{j, k=1}^{r} \alpha_{k} C_{j k}^{j}$ of the adjoint mapping $\operatorname{ad}(\boldsymbol{u})$ if $\operatorname{tr}(\operatorname{ad}(\boldsymbol{u})) \not \equiv 0$ (see Eisenhart [11]). If $\sum_{j=1}^{r} C_{j k}^{j} \not \equiv 0$ for all $k=1, \ldots, r$, there exists the $(r-1)$-dimensional ideal $\{\boldsymbol{w} \in \mathscr{G} \mid \operatorname{tr}(\operatorname{ad}(\boldsymbol{w}))=0\}$ of $\mathscr{G}$.

A (symmetric) bilinear form

$$
\begin{aligned}
\Phi: \mathscr{G} \times \mathscr{G} \rightarrow \mathbb{R} ;\left(\boldsymbol{u}=\sum_{j=1}^{r} \alpha_{j} \boldsymbol{v}_{j}, \boldsymbol{w}=\sum_{k=1}^{r} \beta_{k} \boldsymbol{v}_{k}\right) \\
\mapsto \Phi(\boldsymbol{u}, \boldsymbol{w}):=\sum_{j, k=1}^{r} \alpha_{j} \widetilde{\Phi}_{j k} \beta_{k},
\end{aligned}
$$

where $\left(\tilde{\Phi}_{j k}\right)$ denotes a real (symmetric) matrix, is called invariant (relative to the group $\operatorname{Int}(\mathscr{G})$ ) if

$$
\Phi(\operatorname{Ad}(g)(u), \operatorname{Ad}(g)(w))=\Phi(u, w)
$$

holds for any inner automorphism $\operatorname{Ad}(g) \in \operatorname{Int}(\mathscr{G})$ and for any $\boldsymbol{u}, \boldsymbol{w} \in \mathscr{G}$. Analogously to the invariant linear forms it follows for an invariant bilinear form $\Phi$ and two conjugate vector fields $\boldsymbol{u}, \boldsymbol{w} \in \mathscr{G}$

$$
\begin{aligned}
\Phi(w= & \left.\frac{1}{a} \operatorname{Ad}(g)(u), w=\frac{1}{a} \operatorname{Ad}(g)(u)\right) \\
& =\frac{1}{a^{2}} \Phi(\operatorname{Ad}(g)(u), \operatorname{Ad}(g)(u))=\frac{1}{a^{2}} \Phi(u, u),
\end{aligned}
$$

where $\operatorname{Ad}(g) \in \operatorname{Int}(\mathscr{G})$ and $a \in \mathbb{R}$ are a proper inner automorphism and a proper nonzero real number, respectively. Hence the signs of the terms $\Phi(\boldsymbol{u}, \boldsymbol{u})$ and $\Phi(\boldsymbol{w}, \boldsymbol{w})$ are equal if $\boldsymbol{w}$ is conjugate to $\boldsymbol{u}$. All the vectors $\boldsymbol{u} \in \mathscr{G}$ are separated into the three nonintersecting classes $\Phi(\boldsymbol{u}, \boldsymbol{u})<0, \Phi(\boldsymbol{u}, \boldsymbol{u})=0$ and $\Phi(\boldsymbol{u}, \boldsymbol{u})>0$ for any invariant bilinear form $\Phi$. Another remarkable property of an invariant bilinear form $\Phi$ is the fact that with its help an ideal of $\mathscr{G}$ can be given: The set $\mathscr{H}_{\Phi}:=$ $\{\boldsymbol{w} \in \mathscr{G} \mid \Phi(\boldsymbol{u}, \boldsymbol{w})=0 \forall \boldsymbol{u} \in \mathscr{G}\}$ is an ideal in $\mathscr{G}$. An infinitesimal criterion of invariance of the bilinear mapping
$\Phi: \mathscr{G} \times \mathscr{G} \rightarrow \mathbb{R}$ is

$\Phi(\operatorname{ad}(u)(v), \boldsymbol{w})+\Phi(\boldsymbol{v}, \operatorname{ad}(u)(\boldsymbol{w}))=0$

$$
\text { for any } \boldsymbol{u}, \boldsymbol{v}, \boldsymbol{w} \in \mathscr{G}
$$

(see Ovsiannikov [1]). The Killing form $K_{\mathscr{G}}$ is such an invariant bilinear form if $K_{\mathscr{G}} \not \equiv 0$, and the set

$$
\begin{aligned}
\mathscr{H}_{\boldsymbol{K}_{\mathscr{G}}} & :=\left\{\boldsymbol{w} \in \mathscr{G} \mid K_{\mathscr{G}}(\boldsymbol{u}, \boldsymbol{w})=0 \quad \forall \boldsymbol{u} \in \mathscr{G}\right\} \\
& =\left\{\boldsymbol{w}=\sum_{j=1}^{r} \beta_{j} \boldsymbol{v}_{j} \in \mathscr{G} \mid\left(\beta_{1}, \ldots, \beta_{r}\right) K^{\mathscr{G}}=0\right\}
\end{aligned}
$$

is an ideal of $\mathscr{G}$ (see Eisenhart [11]). We use (10) in the form

$$
\Phi^{\mathscr{G}}(\operatorname{ad}(u)(w), w)=0 \quad \text { for any } \quad u, w \in \mathscr{G}
$$

to determine the common invariant symmetric bilinear form $\Phi^{\mathscr{G}}$ of $\mathscr{G}$. If $\Phi^{\mathscr{G}} \not \equiv 0, \Phi^{\mathscr{G}}$ depends on some free elements of the corresponding matrix $\left(\tilde{\Phi}_{i j}^{\mathscr{G}}\right)$, say $b_{1}, \ldots, b_{q} \in \mathbb{R}(q \in \mathbb{N} ; q \leq r(r-1) / 2)$, and $\Phi^{\mathscr{G}}$ is a real linear combination $\sum_{j=1}^{q} b_{j} \Phi^{(j)}$, where the symmetric matrices corresponding to these invariant bilinear forms $\Phi^{(1)}, \ldots, \Phi^{(q)}$ have fixed real elements. Then we consider for an arbitrary $\boldsymbol{u} \in \mathscr{G}$ the expression $\Phi^{\mathscr{G}}(\boldsymbol{u}, \boldsymbol{u})$ $=\sum_{j=1}^{q} b_{j} \Phi^{(j)}(\boldsymbol{u}, \boldsymbol{u})$, where any expression $\Phi^{(j)}(\boldsymbol{u}, \boldsymbol{u})$ is said to be a bilinear invariant of $\boldsymbol{u}$ (relative to the group Int $(\mathscr{G})$ ). Since any binom in the linear invariants of $\boldsymbol{u}$ is a bilinear invariant of $\boldsymbol{u}$, it is sufficient to determine the linear invariants of $\boldsymbol{u}$ using the common invariant bilinear form $\Phi^{\mathscr{G}}$. Since the Killing form $K_{\mathscr{G}}$ is an invariant bilinear form, the Casimir polynomial ${ }^{4}$ $C_{\mathscr{G}}: \mathscr{G} \rightarrow \mathbb{R} ; \boldsymbol{u} \mapsto C_{\mathscr{G}}(\boldsymbol{u}):=K_{\mathscr{G}}(\boldsymbol{u}, \boldsymbol{u})$ leads to the bilinear invariant $C_{\mathscr{G}}(\boldsymbol{u})$ of $\boldsymbol{u} \in \mathscr{G}$ if $C_{\mathscr{G}} \not \equiv 0$. The maximal number of the functionally independent linear and bilinear invariants of an arbitrary $\boldsymbol{u} \in \mathscr{G}$ is denoted by $\ell^{\prime}(\mathscr{G})$. The total number $\ell_{*}(\mathscr{G})$ of all functionally independent scalar invariants of an arbitrary $\boldsymbol{u} \in \mathscr{G}$ is given by

$$
\ell_{*}(\mathscr{G})=\mathrm{d}(\mathscr{G})-\max _{\boldsymbol{u} \in \mathscr{G}}(\operatorname{rank}(\operatorname{ad}(\boldsymbol{u}))),
$$

and there exists an universal invariant $J_{\mathscr{G}}: \mathscr{G} \rightarrow \mathbb{R}^{\ell *(\mathscr{G})}$ of the group Int $(\mathscr{G})$ (see Ovsiannikov [1]). If $\ell_{*}(\mathscr{G})=$ $\ell^{\prime}(\mathscr{G})$, an universal invariant can be given using a complete set of the determined functionally independent linear and bilinear invariants of $\boldsymbol{u}$. With the help of the Killing polynomial $k_{\mathscr{G}}$ of $\mathscr{G}$, which is the characteristic

\footnotetext{
${ }^{4}$ The notation follows Varadarajan [10].
} 
polynomial of the inner derivation $\operatorname{ad}(\boldsymbol{u})$ for $\boldsymbol{u} \in \mathscr{G}$,

$k_{\mathscr{G}}(\lambda ; \boldsymbol{u}):=\operatorname{det}\left(\lambda \mathbf{i d}_{\mathscr{G}}-\mathbf{a d}(\boldsymbol{u})\right)$

$=\lambda^{r}-\tau_{r-1}(\boldsymbol{u}) \lambda^{r-1}+\tau_{r-2}(\boldsymbol{u}) \lambda^{r-2}-\ldots+(-1)^{\ell} \tau_{\ell}(\boldsymbol{u}) \lambda^{\ell}$,

where id ${ }_{\mathscr{G}}$ denotes the identity of the vector space $\mathscr{G}$ and $\ell>0^{5}$ is the smallest integer $j \geq 0$ such that $\tau_{j}(\boldsymbol{u}) \neq 0$, the $\operatorname{rank}^{6} \ell(\mathscr{G})$ of the Lie algebra $\mathscr{G}$ can be defined as the minimal (algebraic) multiplicity $\ell$ of the eigenvalue 0 of $\operatorname{ad}(\boldsymbol{u})$, obtained when $\boldsymbol{u}$ ranges over the whole space $\mathscr{G}$. Clearly, $1 \leq \ell(\mathscr{G}) \leq r$, and $\ell(\mathscr{G})=r$ if an only if $\mathscr{G}$ is nilpotent (see e.g. Varadarajan [10]), where a Lie algebra $\mathscr{G}$ is said to be nilpotent if the series of ideals $\mathscr{G}_{(0)}:=\mathscr{G}, \mathscr{G}_{(k+1)}:=\left[\mathscr{G}_{(k)}, \mathscr{G}\right]$ with $k=0,1,2, \ldots$, which is called the central series of $\mathscr{G}$, terminates. Since $\max _{\boldsymbol{u} \in \mathscr{G}}(\operatorname{rank}(\operatorname{ad}(\boldsymbol{u}))) \geq \mathrm{d}(\mathscr{G})-\ell(\mathscr{G}), \ell_{*}(\mathscr{G}) \leq \ell(\mathscr{G})$ holds. (Ovsiannikov [1] concludes $\ell_{*}(\mathscr{G})=\ell(\mathscr{G})$ from an inapplicable assumption.)

A vector $\boldsymbol{u} \in \mathscr{G}$, for which the value $\ell$ in (13) is equal to the rank $\ell(\mathscr{G})$, is called a regular element of the Lie algebra $\mathscr{G}$, the others are said to be singular. For an arbitrary regular element $\boldsymbol{u} \in \mathscr{G}$ the real Killing polynomial $k_{\mathscr{G}}(13)$ can be written as

$$
\begin{aligned}
& k_{\mathscr{G}}(\lambda ; \boldsymbol{u})=\operatorname{det}\left(\lambda \mathbf{i d}_{\mathscr{G}}-\mathbf{a d}(\boldsymbol{u})\right) \\
& =\lambda^{\ell(\mathscr{G})} \prod_{j=1}^{R}\left\{\lambda-\lambda_{j}(\boldsymbol{u})\right\}^{v_{j}} \prod_{k=1}^{C}\left\{\left(\lambda-\mu_{k}(\boldsymbol{u})\right)\left(\lambda-\overline{\mu_{k}(\boldsymbol{u})}\right)\right\}^{\hat{v}_{k}},
\end{aligned}
$$

where $\lambda_{1}(\boldsymbol{u}), \ldots, \lambda_{R}(\boldsymbol{u})$ are the distinct real nonzero roots with multiplicities $v_{1}, \ldots, v_{R}$ and $\left(\mu_{1}(\boldsymbol{u}), \overline{\mu_{1}(\boldsymbol{u})}\right), \ldots$, $\left(\mu_{C}(\boldsymbol{u}), \mu_{C}(\boldsymbol{u})\right)$ are the distinct pairs of nonzero conjugate complex roots with multiplicities $\hat{v}_{1}, \ldots, \hat{v}_{C}$ $\left(R, C \in\{0\} \cup \mathbb{N} ; \ell(\mathscr{G})+\sum_{j=1}^{R} v_{j}+2 \sum_{k=1}^{C} \hat{v}_{k}=r\right)$. The nonzero eigenvalues are linear homogeneous functions of the components $\alpha_{1}, \ldots, \alpha_{r}$ of $u=\sum_{j=1}^{r} \alpha_{j} v_{j} \in \mathscr{G}$ (see e.g. Eisenhart [11]), that is, for any nonzero real number $a$ the eigenvalues of $\operatorname{ad}(a \boldsymbol{u})$ are $\lambda_{j}(a \boldsymbol{u})=a \lambda_{j}(\boldsymbol{u})$ and $\mu_{k}(a \boldsymbol{u})=a \mu_{k}(\boldsymbol{u})(j=1, \ldots, R ; k=1, \ldots, C)$. Both the real and the imaginary part ${ }^{7}$ of any nonzero eigenvalue are also real invariants of $\boldsymbol{u}$ and can be written as functions of the components of the universal invariant $J_{\mathscr{G}}$ (see Ovsiannikov [1]). If $\lambda_{j}(\boldsymbol{u})$ is a fixed real nonzero root and the set $\mathscr{H}_{\lambda_{j}}:=\left\{\boldsymbol{w} \in \mathscr{G} \mid \lambda_{j}(\boldsymbol{w})=0\right\}$ is a Lie subalgebra of $\mathscr{G}$ of dimensionality d $\left(\mathscr{H}_{\lambda_{j}}\right)>1$, it is possible to determine the (bi-)linear invariants of the vector field $\boldsymbol{w} \in \mathscr{H}_{\lambda_{j}}$ relative to $\operatorname{Int}\left(\mathscr{H}_{\lambda_{j}}\right)$ in the specified

\footnotetext{
${ }^{5}$ As $\operatorname{ad}(\boldsymbol{u})(\boldsymbol{u})=0$ for any $\boldsymbol{u} \in \mathscr{G}$, it follows $\ell>0$.

${ }^{6}$ Some authors, e.g. Eisenhart [11], define the rank of $\mathscr{G}$ as $\mathrm{d}(\mathscr{G})-\ell(\mathscr{G})=r-\ell(\mathscr{G})$.

$\operatorname{Re}(\xi)(\operatorname{Im}(\xi))$ denotes the real (imaginary) part of the complex number $\xi \in \mathbb{C}$.
}

way. $\mathscr{H}_{\lambda_{j}}$ is an ideal of $\mathscr{G}$ if the eigenvalue $\lambda_{j}(\boldsymbol{u})$ is a linear invariant of $\boldsymbol{u}$ or $\left(\lambda_{j}(\boldsymbol{u})\right)^{2}$ is a positive definite invariant bilinear form in the components of $\boldsymbol{u}$. If $\mathscr{H}_{\mu_{k}}:=\left\{\boldsymbol{w} \in \mathscr{G} \mid \mu_{k}(\boldsymbol{w})=0\right\}=\mathscr{H}_{\mu_{k}}$ is a real subalgebra of $\mathscr{G}$ with $\mathrm{d}\left(\mathscr{H}_{\mu_{k}}\right)>1$ for the fixed pair $\left(\mu_{k}(\boldsymbol{u}), \overline{\left.\mu_{k}(\boldsymbol{u})\right)}\right.$ of conjugate complex eigenvalues, one can calculate also the (bi-)linear invariants of $\boldsymbol{w} \in \mathscr{H}_{\mu_{k}}$ relative to $\operatorname{Int}\left(\mathscr{H}_{\mu_{k}}\right)$ in an analogous way. $\mathscr{H}_{\mu_{k}}$ is an ideal of $\mathscr{G}$ if both $\left(\operatorname{Re}\left(\mu_{k}(\boldsymbol{u})\right)\right)^{2}$ and $\left(\operatorname{Im}\left(\mu_{k}(\boldsymbol{u})\right)\right)^{2}$ are positive definite invariant bilinear forms in the components of $\boldsymbol{u}$. If the sets $\mathscr{H}_{\lambda_{j}}(j=1, \ldots, R)$ and $\mathscr{H}_{\mu_{k}}(k=1, \ldots, C)$ are ideals of $\mathscr{G}$, then for any intersection of the type $\mathscr{H}_{\lambda_{i}} \cap \mathscr{H}_{\lambda_{j}}$, $\mathscr{H}_{\mu_{k}} \cap \mathscr{H}_{\mu_{l}}, \mathscr{H}_{\lambda_{j}} \cap \mathscr{H}_{\mu_{k}}(j \neq \mathrm{i}=1, \ldots, R ; k \neq l=1, \ldots, C)$ and so on, which are also ideals of $\mathscr{G}$, the (bi-)linear invariants of a vector field $\boldsymbol{w}$ in the considered intersection can be calculated. Then the intersection $\mathscr{K}=\bigcap_{j=1}^{R} \mathscr{H}_{\lambda_{j}} \cap \bigcap_{k=1}^{C} \mathscr{H}_{\mu_{k}}$ forms a nilpotent ideal in $\mathscr{G}$, since $\mathrm{d}(\mathscr{K})=\ell(\mathscr{K})$ holds. For any subalgebra $\mathscr{H}$ of $\mathscr{G}$ with dimensionality $\mathrm{d}(\mathscr{H})>1$ the above procedure to determine (bi-)linear invariants of the vector in $\mathscr{H}$ and its subalgebras can be applied as well. A (bi-)linear invariant of a vector field $\boldsymbol{w}$ in $\mathscr{H}$ relative to $\operatorname{Int}(\mathscr{H})$ is an invariant relative to $\operatorname{Int}(\mathscr{G})$ if it coincides with a (bi-)linear invariant of $\boldsymbol{w}$ relative to $\operatorname{Int}(\mathscr{G})$. Therefore the other invariants of $\boldsymbol{w} \in \mathscr{H}$ may be changed by the full adjoint action.

\section{2. "Construction" of Optimal Systems of Solvable Lie Subalgebras}

To determine the optimal system $\Theta_{1}^{\mathscr{G}}$ of solvable one-dimensional subalgebras of the $r$-dimensional Lie algebra $\mathscr{G}$ one starts with the determination of the center $\mathscr{Z}(\mathscr{G})$. (This can be done by annulling all the elements of the matrix $\left(\sum_{l=1}^{r} C_{k l}^{j} \alpha_{l}\right)$ of the adjoint mapping ad $(\boldsymbol{u})$ with $\boldsymbol{u}=\sum_{j=1}^{r} \alpha_{j} \boldsymbol{v}_{j}$ (see the decription of the program OPTSYS).) As remarked above, the central elements generate ideals which cannot be changed by any inner automorphism. During the construction of optimal subalgebraic systems they can be included as a direct term in any subalgebra of the Lie algebra $\mathscr{G}$. It follows that if the optimal systems are known for the factor algebra $\mathscr{G} / \mathscr{Z}(\mathscr{G})$, then they can be considered as known of the entire Lie algebra $\mathscr{G}$. Therefore it is sufficient to consider only Lie algebras with null centers (see Ovsiannikov [1]). 
In what follows, we describe methods for "constructing" an optimal system $\Theta_{1}^{\mathscr{G}}$, under the assumption that there exists a decomposition $\mathscr{G}=\mathscr{Z}(\mathscr{G}) \oplus \widetilde{\mathscr{G}}$ of $\mathscr{G}$ with a not necessarily nonnull center. We choose a basis $\left\{\boldsymbol{v}_{1}, \ldots, \boldsymbol{v}_{r}\right\}$ of $\mathscr{G}$ such that the subalgebra $\tilde{\mathscr{G}} \subset \mathscr{G}$ and the center $\mathscr{Z}(\mathscr{G})$ are spanned by the vectors $\boldsymbol{v}_{1}, \ldots, \boldsymbol{v}_{\tilde{r}}$ and $\boldsymbol{v}_{\tilde{r}+1}, \ldots, \boldsymbol{v}_{\boldsymbol{r}}$, respectively. First we determine the optimal system $\Theta_{1}^{\widetilde{G}}$ for the ideal $\widetilde{G}$ with null center. The two one-dimensional Lie subalgebras $\mathscr{H}(\tilde{\boldsymbol{u}}) \subset \tilde{\mathscr{G}}$ and $\mathscr{K}(\tilde{\boldsymbol{w}}) \subset \tilde{\mathscr{G}}$ with $\tilde{\boldsymbol{u}}=\sum_{j=1}^{\tilde{r}} \alpha_{j} \boldsymbol{v}_{j}$ and $\tilde{\boldsymbol{w}}=\sum_{j=1}^{\tilde{r}} \beta_{j} \boldsymbol{v}_{j}\left(\alpha_{j}, \beta_{j} \in \mathbb{R} ; j=1, \ldots, \tilde{r}\right)$ are conjugate if and only if there are an $\operatorname{Ad}(g) \in \operatorname{Int}(\mathscr{G})$ and a real number $a \neq 0$ such that $\tilde{\boldsymbol{w}}=(1 / a) \operatorname{Ad}(g)(\tilde{\boldsymbol{u}})$. Therefore we consider an arbitrary $\tilde{\boldsymbol{u}} \in \widetilde{\mathscr{G}}$ and choose for $a=1$ a proper inner automorphism $\operatorname{Ad}(g)$ (see (5)), where the parameters $\varepsilon_{1}, \ldots, \varepsilon_{\tilde{r}}$ depend on $\left(\alpha_{1}, \ldots, \alpha_{\tilde{r}}\right)$ such that as much coefficients $\beta_{1}, \ldots, \beta_{\tilde{r}}$ vanish as possible. (None of the parameters $\varepsilon_{\tilde{r}+1}, \ldots, \varepsilon_{r}$ appears in $\operatorname{Ad}(g)$, since $\operatorname{Ad}\left(\exp \left(\varepsilon_{j} \boldsymbol{v}_{j}\right)\right)$ equals $\operatorname{id}_{\mathscr{g}}$ for $j=\tilde{r}+1, \ldots, r$ (see (3)).) This permits the choice of the simplest representative of each class of conjugate subalgebras to which the vector $\tilde{\boldsymbol{u}}$ belongs. At this, $\tilde{\boldsymbol{u}}$ has to range over the whole subalgebra $\widetilde{\mathscr{G}}$, where the various alternative cases give the classes of conjugate one-dimensional subalgebras and, from them, an optimal subalgebraic system $\Theta_{1}^{\widetilde{G}}$. Any linear or bilinear invariant of a vector field $\tilde{\boldsymbol{u}} \in \widetilde{\mathscr{G}}$ places restrictions on how far one can expect to "simplify" $\tilde{\boldsymbol{w}}=\operatorname{Ad}(g)(\tilde{\boldsymbol{u}}) \in \widetilde{\mathscr{G}}, \operatorname{since} \varphi(\tilde{\boldsymbol{w}})=\varphi(\tilde{\boldsymbol{u}})$ and $\Phi(\tilde{\boldsymbol{w}}, \tilde{\boldsymbol{w}})=\Phi(\tilde{\boldsymbol{u}}, \tilde{\boldsymbol{u}})$ holds for any invariant linear form $\varphi: \tilde{G} \rightarrow \mathbb{R}$ and any invariant bilinear form $\Phi: \widetilde{\mathscr{G}} \times \widetilde{\mathscr{G}} \rightarrow \mathbb{R}$, respectively. It $\tilde{\boldsymbol{u}}$ is a regular element of $\widetilde{\mathscr{G}}$, we proved that there exists a conjugate regular vector field $\tilde{\boldsymbol{w}}$ such that all the coefficients of $\tilde{\boldsymbol{w}}$ which are functionally independent of the invariants of $\tilde{\boldsymbol{w}}$ relative to $\operatorname{Int}(\tilde{\mathscr{G}})$ vanish. The same holds if $\tilde{\boldsymbol{u}}$ is a regular element of a subalgebra $\tilde{H}$ of $\widetilde{\mathscr{G}}$. If $\mathscr{Z}(\mathscr{G}) \equiv 0$, then an optimal system $\Theta_{1}^{\mathscr{G}}$ can be "constructed" in the named way, since $\widetilde{\mathscr{G}}=\mathscr{G}$ holds. If $\mathscr{Z}(\mathscr{G}) \not \equiv 0$, one chooses for any conjugacy class in $\Theta_{1}^{\widetilde{G}}$ a "simple" representative $\mathscr{H}(\tilde{\boldsymbol{u}})$ with $\tilde{\boldsymbol{u}}=\sum_{j=1}^{\tilde{r}} \alpha_{j} \boldsymbol{v}_{j} \in \tilde{\mathscr{G}}$ and considers the one-dimensional subalgebras of type $\mathscr{K}(\boldsymbol{u})$, where $\boldsymbol{u}=\tilde{\boldsymbol{u}}+\alpha_{\tilde{\boldsymbol{r}}+1} \boldsymbol{v}_{\tilde{r}+1}+\ldots+\alpha_{r} \boldsymbol{v}_{r} \in \widetilde{\mathscr{G}} \oplus \mathscr{Z}(\mathscr{G})$ with free constants $\alpha_{\tilde{r}+1}, \ldots, \alpha_{r}$. Under the full adjoint action the vector field $\boldsymbol{u}$ spanning a subalgebra $\mathscr{K}(\boldsymbol{u})$ may be conjugate to a "simpler" vector field $\boldsymbol{w}=\operatorname{Ad}(g)(\boldsymbol{u})$ for special choices of the coefficients $\alpha_{\tilde{r}+1}, \ldots, \alpha_{r}$. Thus one determines any conjugacy class of one-dimen- sional subalgebras represented by $\mathscr{K}^{\prime}(\boldsymbol{w})$ and add these to a list. Then one proceeds with considering the next member of $\Theta_{1}^{\overparen{S}}$ in the same way, until all members of this optimal system are investigated. The union of all the listed representatives and the one-dimensional subalgebras of $\mathscr{Z}(\mathscr{G})$ gives $\Theta_{1}^{\mathscr{S}}$.

During the "construction" of an optimal system $\Theta_{2}^{\mathscr{F}}$ consisting of conjugacy classes of two-dimensional subalgebras which are solvable, it is possible to assume in advance that one of the basis vectors $\boldsymbol{u}_{1}, \boldsymbol{u}_{2}$ of a two-dimensional subalgebra $\mathscr{K}\left(\boldsymbol{u}_{1}, \boldsymbol{u}_{2}\right)$, which is a member of a conjugacy class in $\Theta_{2}^{\mathscr{G}}$, is taken from the optimal system $\Theta_{1}^{\mathscr{g}}$. Let this vector be $\boldsymbol{u}_{1}$, where $\mathscr{H}\left(\boldsymbol{u}_{1}\right)$ is a representative subalgebra of a conjugacy class in $\Theta_{1}^{\mathscr{G}}$. Corresponding to it, one chooses a nonnull vector $\boldsymbol{u}_{2} \in \operatorname{Nor}\left(\mathscr{H}\left(\boldsymbol{u}_{1}\right)\right) / \mathscr{H}\left(\boldsymbol{u}_{1}\right)$, where the normalizer of a Lie subalgebra $\mathscr{H}$ of $\mathscr{G}$ is defined as $\operatorname{Nor}(\mathscr{H}):=$ $\{\boldsymbol{v} \in \mathscr{G} \mid[\boldsymbol{v}, \boldsymbol{w}] \in \mathscr{H} \forall \boldsymbol{w} \in \mathscr{H}\}$ and is the largest subalgebra of $\mathscr{G}$ which contains $\mathscr{H}$ as an ideal. From lemma 1, the Lie theorem and the definition of $\Theta_{1}^{\mathscr{G}}$ and $\Theta_{2}^{\mathscr{G}}$ follows that a representative of any class in $\Theta_{2}^{\mathscr{G}}$ can be found in this way. Finally the conjugate ones among the listed solvable subalgebras $\mathscr{K}$ have to be striked out except of one representative. The union of the remaining representatives is $\Theta_{2}^{\mathscr{S}}$.

The optimal subsystem $\Theta_{s}^{\mathscr{g}}$ of $s$-dimensional solvable subalgebras for an $r$-dimensional Lie algebra $\mathscr{G}$ can be developed for $2 \leq s \leq r$ by the "method of expansion" (see Ovsiannikov [1]) of the representative members of the optimal subsystem $\Theta_{s-1}^{\mathscr{g}}$ of $(s-1)$-dimensional solvable subalgebras of $\mathscr{G}$ in the following way:

For any $(s-1)$-dimensional solvable subalgebra $\mathscr{H}\left(\boldsymbol{u}_{1}, \ldots, \boldsymbol{u}_{s-1}\right) \subset \mathscr{G}$, which represents a conjugacy class in $\Theta_{s-1}^{\mathscr{s}}$, one chooses a nonnull vector field $u_{s} \in \operatorname{Nor}\left(\mathscr{H}\left(u_{1}, \ldots, u_{s-1}\right)\right) / \mathscr{H}\left(u_{1}, \ldots, u_{s-1}\right)$. Then the set $\left\{\boldsymbol{u}_{1}, \ldots, \boldsymbol{u}_{s-1}, \boldsymbol{u}_{s}\right\}$ is a basis of an $s$-dimensional subalgebra $\mathscr{K}\left(\boldsymbol{u}_{1}, \ldots, \boldsymbol{u}_{s}\right) \subset \mathscr{G}$. If $\mathscr{K}\left(\boldsymbol{u}_{1}, \ldots, \boldsymbol{u}_{s}\right)$ is solvable, it is conjugate to a class of solvable subalgebras in $\Theta_{s}^{\mathscr{G}}$. One can prove that a representative of any class in the optimal subsystem $\Theta_{s}^{\mathscr{S}}$ is found in this way. This follows from lemma 1 , the Lie theorem and the definition of an optimal subalgebraic system in case of solvable subalgebras. Finally the conjugate ones among the listed subalgebras $\mathscr{K}$ have to be striked out except of one representative, and the remaining list gives an optimal subsystem $\Theta_{s}^{\mathscr{G}}$ of solvable $s$-dimensional subalgebras of $\mathscr{G}$.

If these optimal subalgebraic systems $\Theta_{s}^{\mathscr{G}}$ are found, for any member of the corresponding optimal 
systems $\Theta_{s}^{G}$ of $s$-parameter subgroups of $G$, where $s \leq n^{8}$, a reduced system of partial differential equations with $n-s$ independent variables can be derived in the usual way if some conditions are fulfilled (see Olver [4]). Therefore one is interested only in optimal systems of $s$-dimensional subalgebras with $s \leq n$. (In case of $s=n-1$ the reduced systems, if they exist, are systems of ODEs.) A list of the solutions of all the reduced systems corresponding to the conjugacy classes in an optimal system $\Theta_{s}^{G}$ for fixed $s \leq n$ forms the optimal system of similarity solutions.

\subsection{The REDUCE Programs OPTSYS, BINV and NORM}

If the maximal finite-dimensional ${ }^{9}$ Lie point symmetry group $G$ admitted by the considered system of (integro-)differential equations is determined, i.e. the infinitesimal elements are known, a lot of preparing steps have to be done, before one can begin with "constructing" the optimal systems $\Theta_{s}^{\mathscr{G}}$ of solvable subalgebras of the Lie algebra $\mathscr{G}$ of $G$ with the help of the named techniques. Here, some of these necessary but lengthy calculations can be done using computer algebras systems like REDUCE. We developed a REDUCE 3.2 program named OPTSYS, which calculates for given infinitesimal elements, which depend linearly on the $r$ real parameters $c_{1}, \ldots, c_{r}$,

- a basis $\left\{\boldsymbol{v}_{1}, \ldots, \boldsymbol{v}_{r}\right\}$ of the Lie algebra $\mathscr{G}$ and its commutator table,

- the structure constants of $\mathscr{G}$,

- the matrix of the adjoint mapping ad $(\boldsymbol{u}): \mathscr{G} \rightarrow \mathscr{G}$ for $\boldsymbol{u} \in \mathscr{G}$

- the center $\mathscr{Z}(\mathscr{G})$ of $\mathscr{G}$,

- the Killing form $K_{\mathscr{G}}$, the Killing matrix $K^{\mathscr{G}}$, the Casimir polynomial $C_{\mathscr{g}}$,

- the derived algebra $\mathscr{G}^{(1)}$,

- the Killing polynomial $k_{\mathscr{g}}$, from which the rank $\ell(\mathscr{G})$ of $\mathscr{G}$ follows,

- the common invariant symmetric bilinear form $\Phi^{\mathscr{S}}$ using the criterion (11),

- the functionally independent (bi-)linear invariants of $\boldsymbol{u} \in \mathscr{G}$,

- and the common inner automorphism $\operatorname{Ad}(g)=$ $\boldsymbol{A d}\left(\exp \left(\varepsilon_{1} \boldsymbol{v}_{1}\right)\right) \circ \ldots \circ \boldsymbol{A d}\left(\exp \left(\varepsilon_{\boldsymbol{r}} \boldsymbol{v}_{\boldsymbol{r}}\right)\right)$ (under some assumptions on the determining Lie series).

\footnotetext{
${ }^{8} n$ denotes the number of independent variables in (1).

${ }^{9}$ If $G$ is of infinite dimensionality, it is possible to consider its subgroup of maximal finite dimensionality.
}

By applying Cartan's criterion of solvability, OPTSYS proves whether $\mathscr{G}$ is solvable or not. The determination of the roots of $k_{\mathscr{g}}$ using the standard features of REDUCE 3.2 was not successful in any case. Therefore these eigenvalues of the adjoint mapping have to be calculated by hand.

With the help of the program BINV it is possible to calculate the (bi-)linear invariants of a vector field $\boldsymbol{u}$ in a given subalgebra $\mathscr{H}$ of $\mathscr{G}$ relative to the group of the inner automorphisms $\operatorname{Int}(\mathscr{H})$. Here, BINV uses also the criterion (11) of invariance of a bilinear symmetric mapping $\Phi^{\mathscr{H}}: \mathscr{H} \times \mathscr{H} \rightarrow \mathbb{R}$, where the left-hand-side of this criterion is determined by OPTSYS.

The program NORM determines for a given subalgebra $\mathscr{H} \subset \mathscr{G}$ the normalizer of $\mathscr{H}$ using the structure coeffficients of $\mathscr{G}$, which are calculated by OPTSYS. NORM is useful during the "construction" of an optimal system $\Theta_{s}^{\mathscr{G}}$ of solvable $s$-dimensional subalgebras by the method of expansion of the representatives of the distinct conjugacy classes in $\Theta_{s-1}^{\mathscr{G}}$ in case of $2 \leq s \leq r$.

\section{Ideal MHD Equations for Plane Motions}

In Cartesian coordinates and all variables written as dimensionless quantities, the two-dimensional ideal MHD equations for plane motions of a plasma across a perpendicular magnetic field are

$$
\begin{aligned}
& \frac{\mathrm{d} \varrho}{\mathrm{d} t}+\varrho(\vec{\nabla} \cdot \vec{v})=0, \quad \varrho \frac{\mathrm{d} \vec{v}}{\mathrm{~d} t}+\vec{\nabla}\left(\frac{1}{2} R_{H} h^{2}+p\right)=0, \\
& \frac{\mathrm{d} h}{\mathrm{~d} t}+h(\vec{\nabla} \cdot \vec{v})=0, \quad \frac{\mathrm{d} p}{\mathrm{~d} t}+\gamma p(\vec{\nabla} \cdot \vec{v})=0,
\end{aligned}
$$

with the fluid velocity $\vec{v}=v^{x}(x, y, t) \vec{e}_{x}+v^{y}(x, y, t) \vec{e}_{y}$, magnetic field $\vec{H}=h(x, y, t) \vec{e}_{z}$, mass density $\varrho=$ $\varrho(x, y, t)$, pressure $p=p(x, y, t)$, adiabatic exponent $\gamma$, magnetic pressure number $R_{H}=H_{0}^{2} /\left(4 \pi \varrho_{0} v_{0}^{2}\right)$, where $H_{0}, \varrho_{0}$ and $v_{0}$ are reference values of the magnetic field, mass density and fluid velocity, respectively, and the Lagrangian derivative $\frac{\mathrm{d}}{\mathrm{d} t}=\frac{\partial}{\partial t}+\vec{v} \cdot \vec{\nabla}$.

Fuchs and Richter [12] determined the full Lie point symmetry group $G$ with Lie algebra $\mathscr{G}$ admitted by (14). Galas [7] calculated optimal systems of one-, twoand three-dimensional subalgebras of $\mathscr{G}$ using a procedure which coincides with that of Winternitz and coworkers ([13], [14], [15]). 
In this paper we concentrate on the case $\gamma \neq 2$, where the real Lie algebra $\mathscr{G}$ of the Lie point symmetry group $G=\exp (\mathscr{G})$ admitted by (14) is spanned by the following infinitesimal generators:

$\boldsymbol{v}_{1}=\frac{\partial}{\partial x}, \quad \boldsymbol{v}_{2}=\frac{\partial}{\partial y}, \quad \boldsymbol{v}_{3}=\frac{\partial}{\partial t} \quad \boldsymbol{v}_{4}=t \frac{\partial}{\partial x}+\frac{\partial}{\partial v^{x}}$,

$\boldsymbol{v}_{5}=t \frac{\partial}{\partial y}+\frac{\partial}{\partial v^{y}}, \quad \boldsymbol{v}_{6}=y \frac{\partial}{\partial x}-x \frac{\partial}{\partial y}+v^{y} \frac{\partial}{\partial v^{x}}-v^{x} \frac{\partial}{\partial v^{y}}$,

$\boldsymbol{v}_{7}=x \frac{\partial}{\partial x}+y \frac{\partial}{\partial y}+v^{x} \frac{\partial}{\partial v^{x}}+v^{y} \frac{\partial}{\partial v^{y}}-2 \varrho \frac{\partial}{\partial \varrho}$,

$\boldsymbol{v}_{8}=t \frac{\partial}{\partial t}-v^{x} \frac{\partial}{\partial v^{x}}-v^{y} \frac{\partial}{\partial v^{y}}+2 \varrho \frac{\partial}{\partial \varrho}$,

$\boldsymbol{v}_{9}=h \frac{\partial}{\partial h}+2 \varrho \frac{\partial}{\partial \varrho}+2 p \frac{\partial}{\partial p}$.

The vector fields $\boldsymbol{v}_{1}, \ldots, \boldsymbol{v}_{6} \in \mathscr{G}$ express the special Galilei-invariance of (14), and $\boldsymbol{v}_{7}, \boldsymbol{v}_{8}, \boldsymbol{v}_{9} \in \mathscr{G}$ are the infinitesimal generators of scaling groups. The dimension of $\mathscr{G}$ is $r=\mathrm{d}(\mathscr{G})=\operatorname{dim}(\mathscr{G})=9$. The commutator table of the Lie algebra $\mathscr{G}$ is shown in Table 1, from which the structure constants $C_{j k}^{i}(i, j, k=1, \ldots, 9)$ are readable.

Obviously, the center $\mathscr{Z}(\mathscr{G})$ is a one-dimensional subalgebra spanned by $\boldsymbol{v}_{9}$, which only depends on the variables $h, \varrho$ and $p$. Furthermore, Table 1 shows that a basis of the derived Lie algebra $\mathscr{G}^{(1)}:=[\mathscr{G}, \mathscr{G}]$ is the set $\left\{\boldsymbol{v}_{1}, \boldsymbol{v}_{2}, \boldsymbol{v}_{3}, \boldsymbol{v}_{4}, \boldsymbol{v}_{5}\right\}$. Thus $\mathscr{Z}(\mathscr{G}) \cap \mathscr{G}^{(1)}=0$.

For given $\boldsymbol{u}=\sum_{j=1}^{r} \alpha_{j} \boldsymbol{v}_{j} \in \mathscr{G}$ the derivation $\operatorname{ad}(\boldsymbol{u}): \mathscr{G} \rightarrow \mathscr{G}^{(1)}$ maps $\boldsymbol{w}=\sum_{j=1}^{r} \beta_{j}^{j=1} \boldsymbol{v}_{j} \in \mathscr{G}$ to

$$
\begin{aligned}
\operatorname{ad}(\boldsymbol{u}) & (\boldsymbol{w})=\sum_{i, j, k=1}^{r} \alpha_{k} C_{j k}^{i} \beta_{j} \boldsymbol{v}_{i} \\
= & \left(\alpha_{7} \beta_{1}+\alpha_{6} \beta_{2}+\alpha_{4} \beta_{3}-\alpha_{3} \beta_{4}-\alpha_{2} \beta_{6}-\alpha_{1} \beta_{7}\right) \boldsymbol{v}_{1} \\
& +\left(-\alpha_{6} \beta_{1}+\alpha_{7} \beta_{2}+\alpha_{5} \beta_{3}-\alpha_{3} \beta_{5}-\alpha_{1} \beta_{6}-\alpha_{2} \beta_{7}\right) \boldsymbol{v}_{2} \\
& +\left(\alpha_{8} \beta_{3}-\alpha_{3} \beta_{8}\right) v_{3} \\
& +\left(\left(\alpha_{7}-\alpha_{8}\right) \beta_{4}+\alpha_{6} \beta_{5}-\alpha_{5} \beta_{6}-\alpha_{4}\left(\beta_{7}-\beta_{8}\right)\right) v_{4} \\
& +\left(-\alpha_{6} \beta_{4}+\left(\alpha_{7}-\alpha_{8}\right) \beta_{5}+\alpha_{4} \beta_{6}-\alpha_{5}\left(\beta_{7}-\beta_{8}\right)\right) v_{5} .
\end{aligned}
$$

Thus $\max _{\boldsymbol{u} \in \mathscr{G}}(\operatorname{rank}(\operatorname{ad}(\boldsymbol{u})))=5$. The Killing polynomial

\begin{tabular}{|c|c|c|c|c|c|c|c|c|c|c|}
\hline $\boldsymbol{v}_{i}$ & $\frac{\boldsymbol{v}_{j}}{\left[\boldsymbol{v}_{i}, \boldsymbol{v}_{j}\right]}$ & $\boldsymbol{v}_{1}$ & $v_{2}$ & $\boldsymbol{v}_{3}$ & $v_{4}$ & $v_{5}$ & $v_{6}$ & $\boldsymbol{v}_{7}$ & $v_{8}$ & $v_{9}$ \\
\hline & $\boldsymbol{v}_{1}$ & 0 & 0 & 0 & 0 & 0 & $-v_{2}$ & $v_{1}$ & 0 & 0 \\
\hline & $v_{2}$ & 0 & 0 & 0 & 0 & 0 & $\boldsymbol{v}_{1}$ & $v_{2}$ & 0 & 0 \\
\hline & $v_{3}$ & 0 & 0 & 0 & $v_{1}$ & $\boldsymbol{v}_{2}$ & 0 & 0 & $\boldsymbol{v}_{3}$ & 0 \\
\hline & $v_{4}$ & 0 & 0 & $-v_{1}$ & 0 & 0 & $-v_{5}$ & $v_{4}$ & $-v_{4}$ & 0 \\
\hline & $v_{5}$ & 0 & 0 & $-\boldsymbol{v}_{2}$ & 0 & 0 & $\boldsymbol{v}_{4}$ & $\boldsymbol{v}_{5}$ & $-v_{5}$ & 0 \\
\hline & $v_{6}$ & $\boldsymbol{v}_{2}$ & $-v_{1}$ & 0 & $v_{5}$ & $-v_{4}$ & 0 & 0 & 0 & 0 \\
\hline & $\boldsymbol{v}_{7}$ & $-v_{1}$ & $-v_{2}$ & 0 & $-v_{4}$ & $-v_{5}$ & 0 & 0 & 0 & 0 \\
\hline & $v_{8}$ & 0 & 0 & $-v_{3}$ & $v_{4}$ & $v_{5}$ & 0 & 0 & 0 & 0 \\
\hline & $v_{9}$ & 0 & 0 & 0 & 0 & 0 & 0 & 0 & 0 & 0 \\
\hline
\end{tabular}
$k_{\mathscr{g}}$ has maximal 5 nonzero roots, the simple eigenvalues $\lambda_{1}(\boldsymbol{u})=\alpha_{8}, \mu_{1}(\boldsymbol{u})=\alpha_{7}-\alpha_{8}+i \alpha_{6}, \overline{\mu_{1}(\boldsymbol{u})}, \mu_{2}(\boldsymbol{u})$

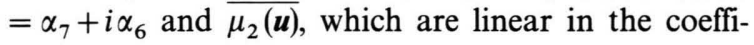

Table 1. The commutator table of the solvable Lie algebra $\mathscr{G}$ for $\gamma \neq 2$.

cients of an regular element $\boldsymbol{u}$ in $\mathscr{G}$. Since $\mathrm{d}(\mathscr{G})=9$, the rank of $\mathscr{G}$ is $\ell(\mathscr{G})=4$ and there must be $\ell_{*}(\mathscr{G})=4$ functionally independent scalar invariants of the group of the inner automorphisms. The computation of the Killing form $K_{\mathscr{g}}(\boldsymbol{u}, \boldsymbol{w}):=\operatorname{tr}(\operatorname{ad}(\boldsymbol{u}) \circ \operatorname{ad}(\boldsymbol{w}))$ and the Casimir polynomial $C_{\mathscr{g}}(\boldsymbol{u}):=K_{\mathscr{g}}(\boldsymbol{u}, \boldsymbol{u})$ gives $K_{\mathscr{g}}(\boldsymbol{u}, \boldsymbol{w})$ $=-4 \alpha_{6} \beta_{6}+2 \alpha_{7} \beta_{7}+2\left(\alpha_{7}-\alpha_{8}\right)\left(\beta_{7}-\beta_{8}\right)+\alpha_{8} \beta_{8}$ and

$$
\begin{aligned}
C_{\mathscr{G}}(\boldsymbol{u})=\lambda_{1}^{2}(\boldsymbol{u}) & +2 \sum_{j=1}^{2} \operatorname{Re}\left(\mu_{j}^{2}(\boldsymbol{u})\right) \\
= & -4 \alpha_{6}^{2}+2 \alpha_{7}^{2}+2\left(\alpha_{7}-\alpha_{8}\right)^{2}+\alpha_{8}^{2} .
\end{aligned}
$$

Consequently, it follows by applying Cartan's criterion of solvability that $\mathscr{G}$ is a solvable Lie algebra. Thus the radical $\mathscr{R}(\mathscr{G})$ of $\mathscr{G}$, i.e. the maximal solvable ideal of $\mathscr{G}$, is $\mathscr{G}$ itself.

The inner automorphisms

$$
\begin{aligned}
\operatorname{Ad}\left(\exp \left(\varepsilon_{j} \boldsymbol{v}_{j}\right)\right):=\exp \left(\operatorname{ad}\left(\varepsilon_{j} \boldsymbol{v}_{j}\right)\right): \mathscr{G} & \rightarrow \mathscr{G} \\
\left(\varepsilon_{j} \in \mathbb{R} ; j\right. & =1, \ldots, 9)
\end{aligned}
$$

can be computated as a Lie series in the usual way and are listed in Table 2. The common element of the group $\operatorname{Int}(\mathscr{G})$ is

$$
\begin{aligned}
\operatorname{Ad}(g= & \left.\exp \left(\varepsilon_{1} \boldsymbol{v}_{1}\right) \cdot \ldots \cdot \exp \left(\varepsilon_{9} \boldsymbol{v}_{9}\right)\right) \\
& =\operatorname{Ad}\left(\exp \left(\varepsilon_{1} \boldsymbol{v}_{1}\right)\right) \circ \ldots \circ \operatorname{Ad}\left(\exp \left(\varepsilon_{9} \boldsymbol{v}_{9}\right)\right),
\end{aligned}
$$

where $\varepsilon_{j} \in \mathbb{R}(j=1, \ldots, 9)$ and $g \in G=\exp (\mathscr{G})$.

By calculating the different symmetric bilinear forms $\Phi: \mathscr{G} \times \mathscr{G} \rightarrow \mathbb{R} ;(\boldsymbol{u}, \boldsymbol{w}) \mapsto \Phi(\boldsymbol{u}, \boldsymbol{w})=\sum_{j, k=1}^{\mathrm{d}(\mathscr{G})} \alpha_{j} \tilde{\Phi}_{j k} \beta_{k}$ which are invariant (relative to the group Int $(\mathscr{G})$ ), we find the 4 linear invariants $\alpha_{6}, \alpha_{7}, \alpha_{8}, \alpha_{9}$ of a vector $\boldsymbol{u}=\sum_{j=1}^{d(\mathscr{G})} \alpha_{j} \boldsymbol{v}_{j} \in \mathscr{G}$. Since the eigenvalues $\lambda_{1}(\boldsymbol{u}), \mu_{1}(\boldsymbol{u})$ and $\mu_{2}(\boldsymbol{u})$ of the adjoint mapping $\operatorname{ad}(\boldsymbol{u})$ are linear in the 
Table 2. Adjoint representation of the Lie group $G$.

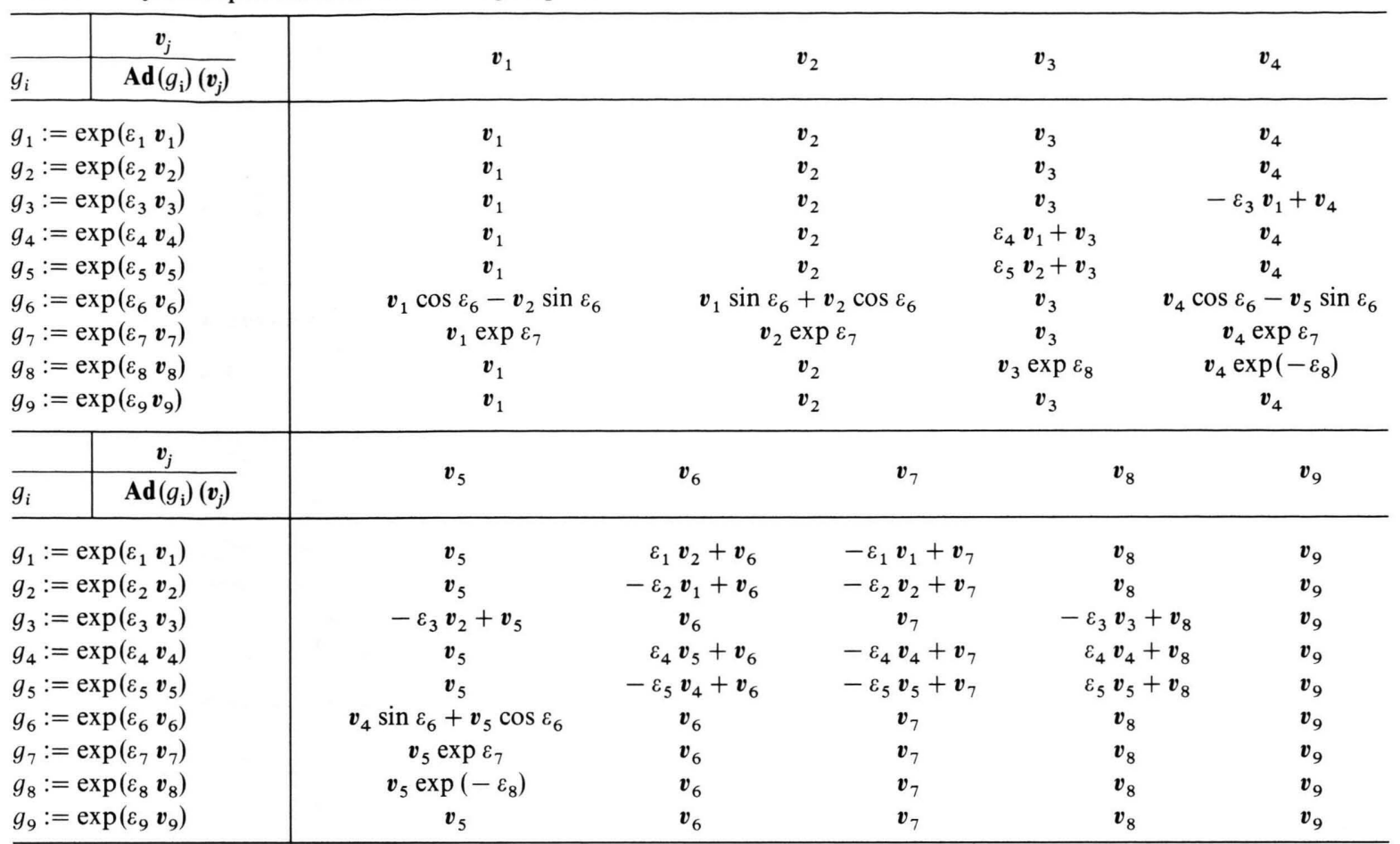

Table 3. (Bi-)linear invariants of a vector $\boldsymbol{u} \in \mathscr{H}$ (relative to $\operatorname{Int}(\mathscr{H})$.

\begin{tabular}{|c|c|c|c|c|c|c|}
\hline & Case & Subalgebra $\mathscr{H}$ & $d(\mathscr{H})$ & $\ell(\mathscr{H})$ & $\ell_{*}(\mathscr{H})$ & Invariants of $\boldsymbol{u}$ \\
\hline 1 & & $\mathscr{H}\left(\boldsymbol{v}_{1}, \ldots, \boldsymbol{v}_{9}\right)=\mathscr{G}$ & 9 & 4 & 4 & $\alpha_{6}, \alpha_{7}, \alpha_{8}, \alpha_{9}$ \\
\hline 2 & $\lambda_{1}(u)=0 \quad\left(\alpha_{8}=0\right)$ & $\mathscr{H}_{\lambda_{1}}\left(\boldsymbol{v}_{1}, \ldots, \boldsymbol{v}_{7}, \boldsymbol{v}_{9}\right)$ & 8 & 4 & 4 & $\alpha_{3}, \alpha_{6}, \alpha_{7}, \alpha_{9}$ \\
\hline 3 & $\begin{array}{l}\mu_{1}(\boldsymbol{u})=0 \\
\left(\alpha_{6}=0, \alpha_{7}=\alpha_{8}\right)\end{array}$ & $\mathscr{H}_{\mu_{1}}\left(v_{1}, \ldots, v_{5}, v_{7}+v_{8}, v_{9}\right)$ & 7 & 4 & 4 & $\begin{array}{l}\alpha_{4}, \alpha_{5} \\
\alpha_{7}=\alpha_{8}, \alpha_{9}\end{array}$ \\
\hline 4 & $\begin{array}{l}\mu_{2}(\boldsymbol{u})=0 \\
\left(\alpha_{6}=\alpha_{7}=0\right)\end{array}$ & $\mathscr{H}_{\mu_{2}}\left(v_{1}, \ldots, v_{5}, v_{8}, v_{9}\right)$ & 7 & 4 & 4 & $\begin{array}{l}\alpha_{1} \alpha_{8}-\alpha_{3} \alpha_{4} \\
\alpha_{2} \alpha_{8}-\alpha_{3} \alpha_{5}, \alpha_{8}, \alpha_{9}\end{array}$ \\
\hline 5 & $\begin{array}{l}\lambda_{1}(\boldsymbol{u})=\mu_{1}(\boldsymbol{u})=\mu_{2}(\boldsymbol{u})=0 \\
\left(\alpha_{6}=\alpha_{7}=\alpha_{8}=0\right)\end{array}$ & $\begin{array}{l}\mathscr{H}\left(\boldsymbol{v}_{1}, \ldots, \boldsymbol{v}_{5}, \boldsymbol{v}_{9}\right) \\
\quad=\mathscr{H}_{\lambda_{1}} \cap \mathscr{H}_{\mu_{1}} \cap \mathscr{H}_{\mu_{2}}\end{array}$ & 6 & 6 & 4 & $\alpha_{3}, \alpha_{4}, \alpha_{5}, \alpha_{9}$ \\
\hline 6 & $\left(\alpha_{3}=\alpha_{6}=\alpha_{7}=\alpha_{8}=0\right)$ & $\mathscr{H}\left(\boldsymbol{v}_{1}, \boldsymbol{v}_{2}, \boldsymbol{v}_{4}, \boldsymbol{v}_{5}, \boldsymbol{v}_{9}\right)$ & 5 & 5 & 5 & $\alpha_{1}, \alpha_{2}, \alpha_{4}, \alpha_{5}, \alpha_{9}$ \\
\hline
\end{tabular}

components of $\boldsymbol{u}$, the sets $\mathscr{H}_{\lambda_{1}}=\left\{\boldsymbol{w} \in \mathscr{G} \mid \lambda_{1}(\boldsymbol{w})=0\right\}$ and $\mathscr{H}_{\mu_{j}}=\left\{\boldsymbol{w} \in \mathscr{G} \mid \mu_{j}(\boldsymbol{w})=0\right\} \quad(j=1,2)$ are ideals of $\mathscr{G}$. For these ideals and for the intersection $\mathscr{H}_{\lambda_{1}} \cap \mathscr{H}_{\mu_{1}} \cap \mathscr{H}_{\mu_{2}} \subset \mathscr{G}$ the invariants of a vector field $\boldsymbol{u}=\sum_{j=1} \alpha_{j} \boldsymbol{v}_{j} \in \mathscr{H}$ relative to the mapping $\operatorname{Ad}(h): \mathscr{H} \rightarrow \mathscr{H}$ with an arbitrary $h \in H=\exp (\mathscr{H})$ are also listed in Table 3 .

The $\ell_{*}(\mathscr{G})=4$ scalar invariants of $\boldsymbol{v}$ relative to the inner automorphism $\mathbf{A d}(g)(g \in G)$ are therefore the real coefficients $\alpha_{6}, \alpha_{7}, \alpha_{8}$ and $\alpha_{9}$. Since $\mathscr{Z}(\mathscr{G}) \cap \mathscr{G}^{(1)}=0$ holds, it is obvious that $\alpha_{9}$ is an invariant. In order to determine an optimal system of one-dimensional subalgebras of $\mathscr{G}$, we start with investigating the Lie algebra $\tilde{\mathscr{G}}$ which is spanned by $\boldsymbol{v}_{1}, \ldots, \boldsymbol{v}_{8}$, i.e. $\tilde{r}=\mathrm{d}(\tilde{\mathscr{G}})$ $=\operatorname{dim}(\tilde{\mathscr{G}})=8$, and has null center. It follows: $\mathscr{G}=\mathscr{Z}(\mathscr{G}) \oplus \tilde{\mathscr{G}}$. Our aim is to find the non unique optimal system $\Theta_{1}^{\mathscr{G}}$ of $\widetilde{\mathscr{G}}$ by separating all one-dimensional subalgebras of $\tilde{G}$ in classes with common invariants for all subalgebras in each class. A represen- 
tative subalgebra $\tilde{\mathscr{H}}$ of each class is assumed to be spanned by $\tilde{\boldsymbol{u}}=\sum_{j=1}^{\tilde{r}} \alpha_{j} \boldsymbol{v}_{j} \in \tilde{\mathscr{H}} \subset \tilde{G}$. We try to "simplify" $\tilde{\boldsymbol{u}}$ by calculating a conjugate $\tilde{\boldsymbol{w}}=\sum_{j=1}^{d(\tilde{\mathscr{G}})} \tilde{\alpha}_{j} \boldsymbol{v}_{j}=\operatorname{Ad}(g)(\tilde{\boldsymbol{u}})$, where as much coefficients $\tilde{\alpha}_{1}, \ldots, \tilde{\alpha}_{8}$ vanish as possible. $\begin{aligned} & \text { First we select } g=\exp \left(\varepsilon_{6} \boldsymbol{v}_{6}\right) \text { and consider } \hat{\boldsymbol{u}}=\sum_{j=1}^{8} \hat{\alpha}_{j} \boldsymbol{v}_{j} \\ = & \operatorname{Ad}\left(\exp \left(\varepsilon_{6} \boldsymbol{v}_{6}\right)\right)(\tilde{u}) . \text { If we choose }\end{aligned}$

$\varepsilon_{6}=\left\{\begin{array}{ccc}-\arctan \left(\alpha_{4} / \alpha_{5}\right) & \text { for } & \alpha_{5} \neq 0 \\ \pi / 2 \operatorname{sign}\left(\alpha_{4}\right) & \text { for } & \alpha_{4} \neq 0 \text { and } \alpha_{5}=0 \\ -\arctan \left(\alpha_{1} / \alpha_{2}\right) & \text { for } & \alpha_{2} \neq 0 \text { and } \alpha_{4}=\alpha_{5}=0 \\ \pi / 2 \operatorname{sign}\left(\alpha_{1}\right) & \text { for } & \alpha_{2}=\alpha_{4}=\alpha_{5}=0\end{array}\right.$ $\tilde{\boldsymbol{u}}$ is conjugate to $\hat{\boldsymbol{u}}=\sum_{j=1}^{3} \hat{\alpha}_{j} \boldsymbol{v}_{j}+\sqrt{\alpha_{4}^{2}+\alpha_{5}^{2}} \boldsymbol{v}_{5}+\sum_{j=6}^{8} \alpha_{j} \boldsymbol{v}_{j}$ or $\hat{\boldsymbol{u}}=\sqrt{\alpha_{1}^{2}+\alpha_{2}^{2}} \boldsymbol{v}_{1}+\alpha_{3} \boldsymbol{v}_{3}+\sum_{j=6}^{8} \alpha_{j} \boldsymbol{v}_{j}$. Therefore we
only need to consider

$\tilde{\boldsymbol{u}}:=\left\{\begin{array}{lr}\alpha_{1} v_{1}+\alpha_{2} v_{2}+\alpha_{3} v_{3}+\alpha_{5} v_{5}+\alpha_{6} v_{6}+\alpha_{7} v_{7}+\alpha_{8} v_{8} \\ \text { or } & \text { with } \alpha_{5}>0 \\ \alpha_{1} v_{1}+\alpha_{3} v_{3}+\alpha_{6} v_{6}+\alpha_{7} v_{7}+\alpha_{8} v_{8} & \text { with } \alpha_{1} \geq 0\end{array}\right.$,

which spans $\tilde{\mathscr{H}}$. We continue with considering the following cases based on the determined invariants of $\tilde{\boldsymbol{u}}$ :

1. $\alpha_{8} \neq 0\left(\lambda_{1}(\tilde{\boldsymbol{u}}) \neq 0\right):$ As Table 3 indicates, $\alpha_{3}$ is no invariant relative to $\operatorname{Int}(\mathscr{G})$ in this case. This fact can easily be verified by applying $\operatorname{Ad}\left(\exp \left(\alpha_{3} / \alpha_{8} \boldsymbol{v}_{3}\right)\right)$ (see Table 2) to $\tilde{\boldsymbol{u}}$. Therefore we choose $\alpha_{3}=0$. By rescaling $\tilde{\boldsymbol{u}}$ we get $\tilde{\boldsymbol{u}}=\alpha_{1} \boldsymbol{v}_{1}+\alpha_{2} \boldsymbol{v}_{2}+\alpha_{5} \boldsymbol{v}_{5}+\alpha_{6} \boldsymbol{v}_{6}$ $+\alpha_{7} v_{7}+v_{8}$, i.e. we set $\alpha_{8}=1$.

1.a. $\alpha_{6} \neq 0\left(\operatorname{Im}\left(\mu_{1 / 2}(\tilde{\boldsymbol{u}})\right) \neq 0\right)$ : The invariants of $\tilde{\boldsymbol{u}}$ are the coefficients $\alpha_{6}, \alpha_{7}, \alpha_{8}=1$. Since $\tilde{u}$ is a regular element of $\widetilde{G}$ and $\alpha_{1}, \alpha_{2}$ and $\alpha_{5}$ are not invariant in this case, the vector $\tilde{\boldsymbol{u}}$ can be "simplified" to $\tilde{\boldsymbol{w}}_{1 a}=$ $\tilde{\alpha}_{6} \boldsymbol{v}_{6}+\tilde{\alpha}_{7} \boldsymbol{v}_{7}+\boldsymbol{v}_{8}$ with $\tilde{\alpha}_{6} \neq 0$.

1.b. $\alpha_{7} \neq \alpha_{6}=0$ and $\alpha_{7} \neq \alpha_{8}=1\left(\operatorname{Re}\left(\mu_{1 / 2}(\tilde{u})\right) \neq 0\right)$ : The nonzero invariants are $\alpha_{7}$ and $\alpha_{8}=1 . \operatorname{Ad}\left(\exp \left(\alpha_{1} v_{1} / \alpha_{7}\right)\right)$ $\circ \boldsymbol{A d}\left(\exp \left(\alpha_{2} \boldsymbol{v}_{2} / \alpha_{7}\right)\right) \circ \boldsymbol{A d}\left(\exp \left(\alpha_{5} \boldsymbol{v}_{5} /\left(\alpha_{7}-1\right)\right)\right)$ maps the regular element $\tilde{\boldsymbol{u}}$ of $\tilde{\mathscr{G}}$ to $\tilde{\boldsymbol{w}}_{1 c}=\tilde{\alpha}_{7} \boldsymbol{v}_{7}+\boldsymbol{v}_{8}$ with $\tilde{\alpha}_{7} \neq 0$ and $\tilde{\alpha}_{7} \neq 1$.

1.c. $\alpha_{6}=0$ and $\alpha_{7}=\alpha_{8}=1\left(\mu_{1}(\tilde{\boldsymbol{u}})=0\right)$ : As case 3 in Table 3 shows, the invariants of $\tilde{\boldsymbol{u}}$ relative to $\operatorname{Int}\left(\tilde{\mathscr{H}}_{\mu_{1}}\left(\boldsymbol{v}_{1}, \ldots, \boldsymbol{v}_{5}, \boldsymbol{v}_{7}+\boldsymbol{v}_{8}\right)\right)$ are $\alpha_{4}=0, \alpha_{5}, \alpha_{7}=\alpha_{8}$ $=1$. We show that the vector $\tilde{\boldsymbol{u}}=\alpha_{1} \boldsymbol{v}_{1}+\alpha_{2} \boldsymbol{v}_{2}$ $+\alpha_{5} v_{5}+v_{7}+v_{8}$ is conjugate to $\hat{\boldsymbol{u}}=\tilde{\alpha}_{5} \boldsymbol{v}_{5}+\boldsymbol{v}_{7}+\boldsymbol{v}_{8}$ with $\tilde{\alpha}_{5} \geq 0 . \tilde{\boldsymbol{u}}$ is mapped to $\hat{\boldsymbol{u}}=\tilde{\alpha}_{5} \boldsymbol{v}_{5}+\boldsymbol{v}_{7}+\boldsymbol{v}_{8}$ by $\operatorname{Ad}\left(\exp \left(\alpha_{1} \boldsymbol{v}_{1}\right)\right) \circ \operatorname{Ad}\left(\exp \left(\alpha_{2} \boldsymbol{v}_{2}\right)\right)$. Further use of
$\operatorname{Ad}\left(\exp \left(\varepsilon_{8} \boldsymbol{v}_{8}\right)\right)$ shows that $\hat{\boldsymbol{u}}$ is conjugate either to $\tilde{\boldsymbol{w}}_{2}=\boldsymbol{v}_{5}+\boldsymbol{v}_{7}+\boldsymbol{v}_{8}$ or to $\tilde{\boldsymbol{w}}_{1 b}=\boldsymbol{v}_{7}+\boldsymbol{v}_{8}$.

1.d. $\alpha_{6}=\alpha_{7}=0\left(\mu_{2}(\tilde{\boldsymbol{u}})=0\right)$ : As case 4 in Table 3 shows, the three invariants of $\tilde{\boldsymbol{u}}$ relative to $\operatorname{Int}\left(\tilde{\mathscr{H}}_{\mu_{2}}\left(\boldsymbol{v}_{1}, \ldots\right.\right.$, $\left.\left.\boldsymbol{v}_{5}, \boldsymbol{v}_{8}\right)\right)$ for $\alpha_{3}=0$ are $\alpha_{1} \alpha_{8}=\alpha_{1}, \alpha_{2} \alpha_{8}=\alpha_{2}$ and $\alpha_{8}=1$. The inner automorphism $\operatorname{Ad}\left(\exp \left(-\alpha_{5} \boldsymbol{v}_{5}\right)\right)$ maps the regular element $\tilde{\boldsymbol{u}}=\alpha_{1} \boldsymbol{v}_{1}+\alpha_{2} \boldsymbol{v}_{2}+\alpha_{5} \boldsymbol{v}_{5}$ $+\boldsymbol{v}_{8}$ of $\tilde{\mathscr{H}}_{\mu_{2}}$ to $\hat{\boldsymbol{u}}=\tilde{\alpha}_{1} \boldsymbol{v}_{1}+\tilde{\alpha}_{2} \boldsymbol{v}_{2}+\boldsymbol{v}_{8}$, which is conjugate either to $\tilde{\boldsymbol{w}}_{3}=\boldsymbol{v}_{1}+\boldsymbol{v}_{8}$ or to $\tilde{\boldsymbol{w}}_{1 d}=\boldsymbol{v}_{8}$.

2. $\alpha_{8}=0\left(\lambda_{1}(\tilde{\boldsymbol{u}})=0\right): \alpha_{3}, \alpha_{6}$ and $\alpha_{7}$ are the invariants of $\tilde{\boldsymbol{u}}$ relative to $\operatorname{Int}\left(\tilde{\mathscr{H}}_{\lambda_{1}}\left(\boldsymbol{v}_{1}, \ldots, \boldsymbol{v}_{7}\right)\right)$ (see case 2 in Table 3).

2.a. $\alpha_{6} \neq 0\left(\operatorname{Im}\left(\mu_{1 / 2}(\tilde{\boldsymbol{u}})\right) \neq 0\right)$ or $\alpha_{7} \neq 0\left(\operatorname{Re}\left(\mu_{1 / 2}(\tilde{\boldsymbol{u}})\right) \neq 0\right)$ : $\alpha_{1}, \alpha_{2}, \alpha_{4}=0$ and $\alpha_{5}$ are no invariants. Further usage of the transformations in Table 2 shows that $\tilde{u}$ is conjugate to one of the vectors $\tilde{\boldsymbol{w}}_{4 / 5}=$ $\pm v_{3}+v_{6}+\tilde{\alpha}_{7} v_{7}, \tilde{\boldsymbol{w}}_{6 / 7}= \pm \boldsymbol{v}_{3}+\boldsymbol{v}_{7}, \tilde{\boldsymbol{w}}_{8}=\boldsymbol{v}_{6}+\tilde{\alpha}_{7} \boldsymbol{v}_{7}$ and $\tilde{\boldsymbol{w}}_{9}=\boldsymbol{v}_{7}$.

2.b. $\alpha_{3} \neq 0$ and $\alpha_{6}=\alpha_{7}=0\left(\mu_{1 / 2}(\tilde{\boldsymbol{u}})=0\right)$ : Case 5 in Table 3 shows that $\alpha_{3}, \alpha_{4}=0$ and $\alpha_{5}$ are the invariants of $\tilde{\boldsymbol{u}} \in \tilde{\mathscr{H}}\left(\boldsymbol{v}_{1}, \ldots, \boldsymbol{v}_{5}\right)=\tilde{\mathscr{H}}_{\lambda_{1}} \cap \widetilde{\mathscr{H}}_{\mu_{1}} \cap \tilde{\mathscr{H}}_{\mu_{2}}$ relative to $\operatorname{Int}(\tilde{\mathscr{H}}) . \tilde{\mathscr{H}}=\mathscr{G}^{(1)}$ is a nilpotent ideal of $\mathscr{G}$. Its center $\mathscr{Z}(\tilde{\mathscr{H}})=\tilde{\mathscr{H}}^{(1)}$ is spanned by $\boldsymbol{v}_{1}$ and $\boldsymbol{v}_{2}$. Since $\alpha_{1}$ and $\alpha_{2}$ are not invariant relative to $\operatorname{Int}(\tilde{\mathscr{G}}), \tilde{\boldsymbol{u}}=$ $\alpha_{1} v_{1}+\alpha_{2} u_{2}+\alpha_{3} v_{3}+\alpha_{5} v_{5}$ with $\alpha_{5} \geq 0$ is conjugate to $\hat{\boldsymbol{u}}=\boldsymbol{v}_{3}+\alpha_{5} / \alpha_{3} \boldsymbol{v}_{5}$, which can be "simplified" to $\tilde{\boldsymbol{w}}_{10}=\boldsymbol{v}_{3}$ for $\alpha_{5}=0$ or to $\tilde{\boldsymbol{w}}_{11 / 12}=\boldsymbol{v}_{3} \pm \boldsymbol{v}_{5}$ for $\alpha_{5} \neq 0$. 2.c. $\alpha_{3}=\alpha_{6}=\alpha_{7}=0$ (see case 6 in Table 3): $\breve{\mathscr{H}}\left(\boldsymbol{v}_{1}, \boldsymbol{v}_{2}, \boldsymbol{v}_{4}, \boldsymbol{v}_{5}\right)$ is a nilpotent ideal in $\mathscr{G}$ and in $\widetilde{\mathscr{G}}$, since $\ell(\tilde{\mathscr{H}})=\mathrm{d}(\tilde{\mathscr{H}})$. Furthermore, this subalgebra $\tilde{\mathscr{H}}$ is abelian. $\tilde{\boldsymbol{u}}=\alpha_{1} \boldsymbol{v}_{1}+\alpha_{2} \boldsymbol{v}_{2}+\alpha_{5} \boldsymbol{v}_{5}$ is conjugate to $\hat{\boldsymbol{u}}=\tilde{\alpha}_{1} \boldsymbol{v}_{1}+\tilde{\alpha}_{5} \boldsymbol{v}_{5}$, which can be "simplified" for $\tilde{\alpha}_{5} \neq 0$ to $\tilde{\boldsymbol{w}}_{13 / 14}= \pm \boldsymbol{v}_{1}+\boldsymbol{v}_{5}$ or $\tilde{\boldsymbol{w}}_{15}=\boldsymbol{v}_{5}$ and for $\tilde{\alpha}_{5}=0$ to $\tilde{\boldsymbol{w}}_{16}=\boldsymbol{v}_{1}$.

So an optimal system $\Theta_{1}^{\widetilde{F}}$ for the subalgebra $\widetilde{\mathscr{G}}\left(\boldsymbol{v}_{1}, \ldots, \boldsymbol{v}_{8}\right)$ is a list of one-dimensional subalgebras, each spanned by one of the vectors $\tilde{\boldsymbol{w}}_{1}, \ldots, \tilde{\boldsymbol{w}}_{16}$.

As mentioned above, the center $\mathscr{Z}(\mathscr{G})$ spanned by $\boldsymbol{v}_{9}$ is a member of $\Theta_{1}^{\mathscr{S}}$, since the center is an invariant one-dimensional subalgebra. To determine $\Theta_{1}^{\mathscr{S}}$ we consider the infinitesimal generators $\boldsymbol{w}_{j}:=\tilde{\boldsymbol{w}}_{j}+\alpha_{9} \boldsymbol{v}_{9} \in \mathscr{G}$ $(j=1, \ldots, 16)$ and $\boldsymbol{w}_{17}:=\boldsymbol{v}_{9}$. (For simplicity we drop the tilde of the coefficients $\tilde{\alpha}_{k}$ of the vectors $\tilde{w}_{j}$.) Then only for $\alpha_{6}=\alpha_{7}=\alpha_{8}=0$ a "simplification" of $\boldsymbol{w}$ is possible. In this case the inner automorphism $\operatorname{Ad}\left(\exp \left(\varepsilon_{6} \boldsymbol{v}_{6}\right)\right) \circ \boldsymbol{A d}\left(\exp \left(\varepsilon_{7} \boldsymbol{v}_{7}\right)\right) \circ \boldsymbol{A d}\left(\exp \left(\varepsilon_{8} \boldsymbol{v}_{8}\right)\right)$ maps the vector $w=\alpha_{1} v_{1}+\alpha_{3} v_{3}+\alpha_{5} v_{5}+\alpha_{9} v_{9}$ to

$$
\begin{aligned}
\hat{\boldsymbol{w}}=\cos \varepsilon_{6} & \exp \left(\varepsilon_{7}\right) \alpha_{1} \boldsymbol{v}_{1}+\exp \left(\varepsilon_{8}\right) \alpha_{3} \boldsymbol{v}_{3} \\
& +\cos \varepsilon_{6} \exp \left(\varepsilon_{7}-\varepsilon_{8}\right) \alpha_{5} \boldsymbol{v}_{5}+\alpha_{9} \boldsymbol{v}_{9} .
\end{aligned}
$$


Table 4. Optimal system $\Theta_{1}^{s}$ of one-dimensional subalgebras.

\begin{tabular}{lll}
$\mathscr{H}\left(\alpha_{6} v_{6}+\alpha_{7} v_{7}+v_{8}+\alpha_{9} v_{9}\right)$ & $\mathscr{H}\left(\boldsymbol{v}_{1}+\boldsymbol{v}_{8}+\alpha_{9} \boldsymbol{v}_{9}\right)$ & $\mathscr{H}\left(\boldsymbol{v}_{1}+\boldsymbol{v}_{9}\right)$ \\
$\mathscr{H}\left(\boldsymbol{v}_{5}+\boldsymbol{v}_{7}+\boldsymbol{v}_{8}+\alpha_{9} \boldsymbol{v}_{9}\right)$ & $\mathscr{H}\left( \pm \boldsymbol{v}_{1}+\boldsymbol{v}_{5}+\boldsymbol{v}_{9}\right)$ & $\mathscr{H}\left( \pm \boldsymbol{v}_{1}+\boldsymbol{v}_{5}\right)$ \\
$\mathscr{H}\left( \pm \boldsymbol{v}_{3}+\boldsymbol{v}_{6}+\alpha_{7} \boldsymbol{v}_{7}+\alpha_{9} \boldsymbol{v}_{9}\right)$ & $\mathscr{H}\left(\boldsymbol{v}_{7}+\alpha_{9} \boldsymbol{v}_{9}\right)$ & $\mathscr{H}\left(\boldsymbol{v}_{9}\right)$ \\
$\mathscr{H}\left(\boldsymbol{v}_{6}+\alpha_{7} \boldsymbol{v}_{7}+\alpha_{9} \boldsymbol{v}_{9}\right)$ & $\mathscr{H}\left(\boldsymbol{v}_{5}+\boldsymbol{v}_{9}\right)$ & $\mathscr{H}\left(\boldsymbol{v}_{5}\right)$ \\
$\mathscr{H}\left( \pm \boldsymbol{v}_{3}+\boldsymbol{v}_{7}+\alpha_{9} \boldsymbol{v}_{9}\right)$ & $\mathscr{H}\left(\boldsymbol{v}_{3} \pm \boldsymbol{v}_{9}\right)$ & $\mathscr{H}\left(\boldsymbol{v}_{3}\right)$ \\
$\mathscr{H}\left(\boldsymbol{v}_{3} \pm \boldsymbol{v}_{5}+\boldsymbol{v}_{9}\right)$ & $\mathscr{H}\left(\boldsymbol{v}_{3}+\boldsymbol{v}_{5}\right)$ & \\
\hline
\end{tabular}

Therefore $\boldsymbol{w}_{10}=\boldsymbol{v}_{3}+\alpha_{9} \boldsymbol{v}_{9}, \quad \boldsymbol{w}_{11 / 12}=\boldsymbol{v}_{3} \pm \boldsymbol{v}_{5}+\alpha_{9} \boldsymbol{v}_{9}$, $\boldsymbol{w}_{13 / 14}= \pm \boldsymbol{v}_{1}+\boldsymbol{v}_{5}+\alpha_{9} \boldsymbol{v}_{9}, \boldsymbol{w}_{15}=v_{5}+\alpha_{9} \boldsymbol{v}_{9}, \boldsymbol{w}_{16}=\boldsymbol{v}_{1}+$ $\alpha_{9} v_{9}$ is conjugate to $w_{10 a / 10 b}=v_{3} \pm v_{9}$ or $w_{10 c}=v_{3}$, $\boldsymbol{w}_{11 a / 12 a}=v_{3} \pm v_{5}+v_{9}$ or $\boldsymbol{w}_{11 b}=v_{3}+v_{5}, \quad \boldsymbol{w}_{13 a / 14 a}=$ $\pm v_{1}+v_{5}+v_{9}$ or $w_{13 b / 14 b}= \pm v_{1}+v_{5}, w_{15 a}=v_{5}+v_{9}$ or $\boldsymbol{w}_{15 b}=\boldsymbol{v}_{5}, \boldsymbol{w}_{16 a}=\boldsymbol{v}_{1}+\boldsymbol{v}_{9}$ or $\boldsymbol{w}_{16 b}=\boldsymbol{v}_{1}$, respectively. The resulting optimal system $\Theta_{1}^{\mathscr{P}}$ is listed in Table 4, where $\mathscr{H}(\boldsymbol{w})$ denotes the subalgebra that is spanned by the vector field $\boldsymbol{w}$. The coefficients $\alpha_{6}, \alpha_{7}, \alpha_{9}$ in Table 4 are arbitrary real numbers. Hence $\mathscr{H}\left(\alpha_{6} \boldsymbol{v}_{6}+\alpha_{7} \boldsymbol{v}_{7}+\boldsymbol{v}_{8}\right.$ $\left.+\alpha_{9} \boldsymbol{v}_{9}\right)$ stands for an infinite number of one-dimensional subalgebras with fixed real values of $\alpha_{6}, \alpha_{7}$, and $\alpha_{9}$. The optimal system $\Theta_{1}^{G}$ for the Lie group $G$ is a list of all one-parameter subgroups $\exp (\mathscr{H}(\boldsymbol{w})) \subset G$, where $\mathscr{H}(\boldsymbol{w})$ represents a class of one-dimensional subalgebras in $\Theta_{1}^{\mathscr{G}}$.

The optimal system of one-dimensional Lie subalgebras in Table 4 coincides with that of Galas [7], who calculated the similarity variables and reduced systems of PDEs which correspond to the members of $\Theta_{1}^{\mathscr{G}}$. An optimal system of two-dimensional subalgebras of $\mathscr{G}$ can be found by the method of expansion of representatives of the conjugacy classes in $\Theta_{1}^{\mathscr{G}}$. Lack of space precludes pursuing this interesting problem here. In [7] Galas determined the optimal systems $\Theta_{2}^{\mathscr{S}}$ and $\Theta_{3}^{\mathscr{S}}$ and the reduced systems of ODEs for $\Theta_{2}^{G}$ and the systems of algebraic equations for $\Theta_{3}^{G}$. Galas and Richter [16] discussed similarity solutions for four members of the optimal system $\Theta_{2}^{G}$ of two- parameter subgroups, which describe plasma expansion into vacuum, shock waves and spiral flows with plasma sources.

\section{Concluding Remarks}

"Optimal systems" of similarity solutions of a given system of nonlinear (integro-)differential equations which admits a finite-dimensional Lie point symmetry group $G$ with its Lie algebra $\mathscr{G}$, are an effective, systematic means of classifying these group-invariant solutions. The problem of finding such optimal systems of similarity solutions leads to that of "constructing" optimal subalgebraic systems for $\mathscr{G}$. At this computeraided calculation of linear and bilinear forms which are invariant relative to the group of the inner automorphisms of $\mathscr{G}$, is practicable and helpful to shorten and to simplify the classification process. If $\mathscr{G}$ is solvable, the construction of optimal subalgebraic systems can be done with the help of the described technique. In addition to the optimal systems of solvable subalgebras of a non solvable Lie algebra $\mathscr{G}$ one has to determine the optimal systems of semisimple subalgebras of $\mathscr{G}$ to "construct" the full optimal systems of $s$-dimensional subalgebras of $\mathscr{G}$ if $s \geq 3$. The available techniques for this classification process will be presented in a following paper, where they will be applied to the eight-dimensional Lie point symmetry group $G$ with its non solvable Lie algebra $\mathscr{G}$ admitted by the three-dimensional Vlasov-Maxwell equations in the non-relativistic case.

\section{Acknowledgement}

I would like to thank not only Prof. Dr. E. W. Richter and F. Galas for many useful discussions, but also S. Zidowitz for his aid in developing and testing the named REDUCE-programs. Also I thank Prof. Dr. R. Löwen, Prof. Dr. G. Janssen, and Dr. Görnitz for talks on Lie group and Lie algebra questions. 
[1] L. V. Ovsiannikov, Group analysis of differential equations, Academic Press, New York 1982.

[2] N. H. Ibragimov, Transformation Groups Applied to Mathematical Physics, D. Reidel Publishing Company, Dordrecht 1985.

[3] G. W. Bluman and J. D. Cole, Similarity Methods for Differential Equations, in: Applied Mathematical Sciences, vol. 13, Springer-Verlag, New York 1974.

[4] P. J. Olver, Applications of Lie Groups to Differential Equations, in: Graduate Texts in Mathematics, vol. 107. Springer-Verlag, New York 1986.

[5] G. W. Bluman and S. Kumei, Symmetries and Differential Equations, in: Applied Mathematical Sciences, vol. 81, Springer-Verlag, New York 1989.

[6] H. Stephani, Differential equations. Their solution using symmetries, Cambridge University Press, Cambridge 1989.

[7] F. Galas, Klassifikation und Berechnung der Ähnlichkeitslösungen für zweidimensionale ideale magnetohydrodynamische Strömungen, Master's thesis, Institut für Mathematische Physik, Technische Universität Car-
olo-Wilhelmina zu Braunschweig, Mendelssohnstraße 3, D-3300 Braunschweig, Oktober 1988.

[8] N. Jacobson, Lie Algebras, Dover Publications, New York 1979.

[9] A. A. Sagle and R. E. Walde, Introduction to Lie Groups and Lie Algebras, Academic Press, New York 1973.

[10] V. S. Varadarajan, Lie Groups, Lie Algebras, and Their Representations, in: Graduate Texts in Mathematics, vol. 102, Springer-Verlag, New York 1984.

[11] L. P. Eisenhart, Continuous Groups of Transformations, Dover Publications, New York 1961.

[12] J. C. Fuchs and E. W. Richter, J. Phys. A: Math. Gen. 20, 3135 (1987).

[13] P. Winternitz and L. Gagnon, J. Phys. A: Math. Gen. 21, 1493 (1988).

[14] P. Winternitz and L.Gagnon, J. Phys. A: Math. Gen. 22, 469 (1989).

[15] P. Winternitz, L. Gagnon, B. Grammaticos, and A. Ramani, J. Phys. A: Math. Gen. 22, 499 (1989).

[16] F. Galas and E. W. Richter, Physica D 50, 297 (1991). 
